\title{
A Runaway Yellow Supergiant Star in the Small Magellanic Cloud*
}

Kathryn F. Neugent ${ }^{1,2}$, Philip Massey ${ }^{1,3}$, Nidia Morrell ${ }^{4}$, Brian Skiff ${ }^{1}$, and Cyril Georgy ${ }^{5}$

\begin{abstract}
We recently discovered a yellow supergiant (YSG) in the Small Magellanic Cloud (SMC) with a heliocentric radial velocity of $\sim 300 \mathrm{~km} \mathrm{~s}^{-1}$ which is much larger than expected for a star in its location in the SMC. This is the first runaway YSG ever discovered and only the second evolved runaway star discovered in a different galaxy than the Milky Way. We classify the star as G5-8 I, and use dereddened broad-band colors with model atmospheres to determine an effective temperature of $4700 \pm 250 \mathrm{~K}$, consistent with what is expected from its spectral type. The star's luminosity is then $\log L / L_{\odot} \sim 4.2 \pm 0.1$, consistent with it being $\mathrm{a} \sim 30 \mathrm{Myr} 9 M_{\odot}$ star according to the Geneva evolution models. The star is currently located in the outer portion of the SMC's body, but if the star's transverse peculiar velocity is similar to its peculiar radial velocity, in $10 \mathrm{Myr}$ the star would have moved $1.6^{\circ}$ across the disk of the SMC, and could easily have been born in one of the SMC's star-forming regions. Based on its large radial velocity, we suggest it originated in a binary system where the primary exploded as a supernovae thus flinging the runaway star out into space. Such stars may provide an important mechanism for the dispersal of heavier elements in galaxies given the large percentage of massive stars that are runaways. In the future we hope to look into additional evolved runaway stars that were discovered as part of our other past surveys.
\end{abstract}

Subject headings: supergiants — Local Group — galaxies: individual (SMC) Magellanic Clouds

\footnotetext{
${ }^{*}$ This paper includes data gathered with the 6.5-m Magellan Telescopes located at Las Campanas Observatory, Chile.

${ }^{1}$ Lowell Observatory, 1400 W Mars Hill Road, Flagstaff, AZ 86001; kneugent@lowell.edu; phil.massey@lowell.edu; bas@lowell.edu

${ }^{2}$ Department of Astronomy, University of Washington, Seattle, WA, 98195

${ }^{3}$ Department of Physics and Astronomy, Northern Arizona University, Flagstaff, AZ, 86011-6010

${ }^{4}$ Las Campanas Observatory, Carnegie Observatories, Casilla 601, La Serena, Chile; nmorrell@lco.cl

${ }^{5}$ Geneva University, Geneva Observatory, CH-1290 Versoix, Switzerland; cyril.georgy@unige.ch
} 


\section{Discovery}

Neugent et al. (2010) conducted a radial velocity study of yellow stars seen in the direction of the Small Magellanic Cloud (SMC) in order to identify its yellow supergiant (YSG) population. As shown in Figure 1, the observed radial velocities are either clustered around $0 \mathrm{~km} \mathrm{~s}^{-1}$ (as expected for foreground yellow dwarfs), or around the SMC's heliocentric radial velocity of $158 \mathrm{~km} \mathrm{~s}^{-1}$ (Richter et al. 1987) as indicated by the black line and expected for SMC yellow supergiants. One star, J01020100-7122208, however, has a heliocentric radial velocity of around $300 \mathrm{~km} \mathrm{~s}^{-1}, 140 \mathrm{~km} \mathrm{~s}^{-1}$ greater than expected. Neugent et al. (2010) don't explicitly comment on this star; at the time we believed it to be a likely short-period binary. However, we have now completed additional observations that rule out this explanation. Instead, this star is the first runaway YSG discovered and the second evolved runaway star discovered in another galaxy.

The concept of runaway massive stars has been around for sixty years. Blaauw (1956a, 1956b) discovered that some OB stars stars have much higher space velocities than other stars in their surroundings. Zwicky (1957) was the first to hypothesize that such stars should exist due to supernovae explosions causing the secondary in a binary system to be shot off into space. However, Gies \& Bolton (1986) showed that the close binary frequency of runaway OB stars was the same as for non-runaways, suggesting that the binary ejection mechanism was not the primary source of runaway stars. There have since been other theories as to how such runaway stars exist including the effects of different dynamical interactions (Leonard \& Duncan 1990) or interactions with massive black holes (Capuzzo-Dolcetta \& Fragione 2015; Fragione \& Capuzzo-Dolcetta 2016). Runaway stars aren't rare, with as many as 50\% of OB stars considered runaways with peculiar (difference between observed and expected) radial velocities larger than $40 \mathrm{~km} \mathrm{~s}^{-1}$ (Gies \& Bolton 1986).

Given that such a large fraction of $\mathrm{OB}$ stars are runaways, it is surprising that there are very few evolved massive stars known to be runaways. Three Galactic red supergiants (RSGs), Betelgeuse, $\mu$ Cep and IRC-10414, are thought to be runaways based upon the presence of bow shocks (Noriega-Crespo et al. 1997, Cox et al. 2012, and Gvaramadze et al. 2013). Evans \& Massey (2015) recently identified a runaway RSG in the Andromeda Galaxy directly from its peculiar velocity. The star we discuss here is the first known runaway YSG anywhere and is only the second known evolved massive star runaway of any kind found in another galaxy. In Section 2 we'll discuss our observations and how we calculated the radial velocities. In Section 3, we'll give an overview of the physical properties of this runaway YSG. In Section 4 we'll discuss our results and conclusions. 


\section{Observations, Reductions, and Radial Velocity Calculation}

As mentioned above, we originally believed that the star's abnormally large radial velocity was due to binary motion. Once we entertained the idea of it being a runaway star we obtained three additional spectra to investigate this possibility.

Our first (discovery) spectrum was obtained using Hydra on the CTIO 4-m Blanco telescope on (UT) 2009 Oct 9. The second spectrum was obtained on (UT) 2017 Aug 16 using the Echelle on the du Pont 2.1-m telescope on Las Campanas in Chile. The spectrum was under-exposed in the blue, as our goal was simply to check on the radial velocity from the Ca II triplet in the far red. Subsequently (2017 Dec 31) we obtained a high signal-tonoise spectrum with MagE on the Las Campanas Baade 6.5-m Magellan telescope for the purposes of spectral classification and radial velocity measurement, plus a shorter exposure the following night simply to confirm the radial velocity. The wavelength ranges, spectral resolution $(\Delta \lambda / \lambda)$, and exposure times are summarized in Table 1, To help with spectral classification we also observed six bright $(V \sim 10-12)$ yellow supergiants in the SMC and LMC, ranging in type from F0 to G8.

The full calibration and reduction details for the Hydra spectrum are given in Neugent et al. (2010). For the MagE spectra, we obtained bias and flat-field exposures during the day, and each program exposure was followed immediately by a comparison arc exposure. We used Jack Baldwin's mtools routines in IRAF1 for the spectral extractions; these routines are available from the Las Campanas website. The standard IRAF echelle reduction tasks were then used for wavelength calibration and flux calibration using spectrophotometric standards. For the duPont data, Milky flats were obtained with the afternoon sky and a diffusor in front of the slit, and the reductions are all performed with IRAF, without intervention of the 'mtools' package.

We measured the radial velocities using two methods: first, by fitting a Gaussian to each of the Ca II triplet lines $(\lambda 8498, \lambda 8542, \lambda 8662)$, and secondly by cross-correlating the Hydra radial velocity standard stars from Neugent et al. (2010) against the new spectra in the region around the CaII triplet. The two methods agreed to within $1 \mathrm{~km} \mathrm{~s}^{-1}$. We give the average heliocentric radial velocities (HRV) of the two methods in Table 1. All four values are consistent with each other, essentially ruling out the possibility that the large peculiar radial velocity is due to motion in a close binary system. A small difference is seen in the values between various telescopes, which we believe is due to minor offsets in

\footnotetext{
${ }^{1}$ IRAF is distributed by the National Optical Astronomy Observatory, which is operated by the Association of Universities for Research in Astronomy (AURA) under a cooperative agreement with the National Science Foundation.
} 
the instrumental velocity zero-points. To investigate this further, we measured the radial velocities of the six MagE spectral standards, five of which have published radial velocities from other sources. As is shown in Table 2, we find that the differences ranged from $-14.0 \mathrm{~km}$

$\mathrm{s}^{-1}$ to $+18.6 \mathrm{~km} \mathrm{~s}^{-1}$, consistent with our assertion that our measurements do not indicate any significant radial velocity variations for our runaway.

For consistency we also remeasured the velocity from our original Hydra spectrum. Much to our chagrin, we discovered that we had originally misapplied the heliocentric correction. This affects all of the individual radial velocities given in Table 1 of that paper by $\sim 11.0 \mathrm{~km}$ $\mathrm{s}^{-1}$, with the previously published values too large. We take the opportunity to reissue the table here, as Table 3 . Note, however, that this does not affect anything else in that paper, as our assignment of membership to the SMC was based upon the distribution of velocities, and those were completely accurate in a relative sense, since the heliocentric corrections for all stars were the same. We also took the opportunity to update the table with revised spectral types that were subsequently published.

\section{Physical Properties}

In this section we use our spectral information and photometry to determine the star's effective temperature and luminosity, allowing us to place the star on the H-R diagram. We can then use evolution models to determine other physical properties, such as approximate mass and age.

\subsection{Spectral Classification}

In Figure 2 we compare the spectrum of our star to six spectral standards, Sk 105 (F0 Ia), Sk 55 (F3 Iab), HD 271182 (F8 0), HD 269953 (G0 0), HD 269723 (G4 0), HD 268757 (G8 0). The two early F stars are SMC members classified by Ardenberg \& Maurice (1977); the others are LMC members classified by Keenan \& McNeil (1989), and considered to be MK standards. (The luminosity class " 0 " is one notch above the Ia designation.) We see immediately that our runaway is of G-type, based both upon the abundance of metal lines (despite the lower metallicity of the SMC compared to that of the LMC), and the weakness of the hydrogen lines. The strength of the G-band argues that the star is closer to type G8 than to G0; we assign a type of G5-8 I. For G stars, the strengths of the Ca II H and K lines provide a crude luminosity indicator, developing wide wings at high luminosities (Kaler 2011), and it is clear from Figure 2 that these lines in our runaway are comparable to those 
of the supergiant standards.

\section{2. $\quad$ Effective Temperature}

The uncertainties in the effective temperature scale for late-type supergiants are nicely discussed in the classic review by Böhm-Vitense (1981). A typical temperature for a G8 I star is 4570-90 K, while a G3 I star would be $4980 \mathrm{~K}$ (Böhm-Viense 1981, Cox 2000). Thus the expected temperature range for our star would be $4500-4800 \mathrm{~K}$, with the caveat that the relationship between spectral type and effective temperature has only been established for solar metallicity stars. Since the metallicity of the SMC is roughly one-quarter solar, we expect that the effective temperature will be slightly cooler, probably by $\sim 100 \mathrm{~K}$, based on our previous modeling experience of RSGs (see e.g., Levesque et al. 2006).

We can refine our estimate of the effective temperature by instead using broad-band photometry and appealing to stellar atmosphere models of the appropriate metallicity. The major uncertainty is what to assume for the reddening. The observed colors are included in Table 3 . According to Cox (2000), the intrinsic $(B-V)_{0}$ of a G5 I star is 1.02, while that of a G8 $\mathrm{I}$ is 1.14 . The observed $B-V$ color is 1.15 , suggesting a color excess in the range $E(B-V)=0.01-0.14$. However, we know that the foreground reddening towards the SMC corresponds to an $E(B-V)=0.04$, while the typical color excess of SMC OB stars is $E(B-V)=0.09$ (Massey et al. 2007). Let us consider then three different values for the reddening, $E(B-V)=0.04$ (minimum, only foreground), 0.09 (typical of OB stars), and 0.14. For the other colors, we adopt the relations between color excesses given by Schlegel et al. (1998), e.g., $E(U-B)=0.72 E(B-V), E(V-K)=2.95 E(B-V)$, and $E(J-K)=0.54 E(J-K)$.

In Table 4 we list the deredded colors, along with the corresponding effective temperatures derived from the updated ATLAS9 models (Castelli \& Kurucz 2003) for a metallicity of [-0.5], corresponding to $0.3 \times$ solar, appropriate for the $\mathrm{SMC} 2$. The range of reddening we consider changes the derived temperature by only $100 \mathrm{~K}$. There is a larger difference $(500 \mathrm{~K})$ depending upon which color index we use 3 . We adopt an average temperature $4700+/-250$,

\footnotetext{
${ }^{2}$ http://www.user.oats.inaf.it/castelli/colors/BCP/BCP_m05k2odfnew.dat

${ }^{3}$ To compute the temperature from the models we applied simple linear interpolation between the colors and temperatures. Using the models required us to adopt a value for the surface gravity, $g$. We had to perform this process iteratively, as the surface gravity depends upon the adopted luminosity to compute the stellar radius, and luminosity depends upon the temperature for the bolometric correction. We assumed a mass of $9 M_{\odot}$ based upon the stellar evolution tracks described in the next section. This process converged
} 
in good agreement with the temperature we obtained via the spectral type. Note that the two methods are essentially independent of each other.

We adopt an absolute visual magnitude of the star $M_{V}=-5.4 \pm 0.2$, based upon a distance of the SMC of $59 \mathrm{kpc}$ (van den Bergh 2000). The uncertainty represents the difference in the correction for extinction based upon the amount of reddening assumed, with $A_{V}=3.1 E(B-V)$. The bolometric correction is then $-0.4 \pm 0.2$ based on the ATLAS9 models, with the error corresponding to the uncertainty in temperature. Thus the star's luminosity is $\log L / L_{\odot}=4.2 \pm 0.1$. We summarize the physical properties in Table 5 .

\subsection{H-R Diagram}

In Figure 3 we show the star's location in the H-R diagram and include the latest version of the Geneva evolution tracks. These have been interpolated to $z=0.004$ (appropriate for the SMC) using the grid of models from Georgy et al. (2013). We see that the luminosity and effective temperature of our runaway corresponds to a star with an initial mass of about $9 M_{\odot}$. The tracks were computed using the assumption of an initial equatorial rotational velocity that is $40 \%$ of the critical break-up speed, which is probably the most representative (see discussion in Ekström et al. 2012 and Georgy et al. 2013). However, we show by the dotted $9 M_{\odot}$ track the effect of a $1.5 \times$ lower rotational velocity. The age associated with this stage is about $30 \mathrm{Myr}$, regardless of the initial assumptions. It must be noted, however, that the expulsion mechanism behind this star becoming a runaway could have altered its evolutionary background and thus the age and mass we are able to predict.

\section{Discussion and Conclusions}

We briefly considered the possibility that this was was a foreground object: a very metal poor star out in the Galactic halo, for instance, could have a very high space velocity. Once we obtained the blue/optical spectrum described here, though, we could rule out this interpretation, as such a metal-poor object would show almost no spectral features other than the Balmer lines, the G-band, and $\mathrm{H}$ and $\mathrm{K}$, while our optical spectrum discussed above shows numerous metal lines. The remote possibility that this was a foreground dwarf with an extraordinary radial velocity could also be dismissed from the wide wings of of the $\mathrm{H}$ and $\mathrm{K}$ lines, indicating high luminosity, as discussed in Section 3.

quickly, with a $\log g$ of 0.8 [cgs]. 
The spatial location of the runway YSG is shown in Figure 4. It is not located within the central portion of the SMC and is instead out on the edge of the galaxy, far from any regions of current star formation. We posed the following question: If the star's tangential motion is the same as its peculiar radial velocity, how far would it move during its lifetime? We used $10 \mathrm{Myr}$ as a ball-park estimate for the time involved, since we do not know how long it has been traveling (the star's age is $30 \mathrm{Myr}$ ); in that period of time it would have moved 1.6 degrees! This distance is shown in Figure团as the large red circle. It clearly encompasses the central portion of the SMC suggesting that this star could have been ejected from the body of the galaxy as an unevolved OB star. Note, however, that the kinematics of the SMC is quite complicated. Stanimirovic et al. (2004) found that there is a velocity gradient throughout the galaxy with the northeast quadrant (close to where this star is located) rotating up to $10 \mathrm{~km} \mathrm{~s}^{-1}$ faster than the SMC's average heliocentric radial velocity. Thus, it is possible that this star's peculiar velocity is smaller than expected, but by no more than $10 \mathrm{~km} \mathrm{~s}^{-1}$ (see Figure 3 in Stanimirovic et al. 2004).

We suspect that the YSG began in a binary system and was flung out into space when the primary star went supernova. Gies \& Bolton (1986) concluded that supernova explosions are not the primary mechanism behind runaway stars but most runaway stars have peculiar velocities on the order of $40-80 \mathrm{~km} \mathrm{~s}^{-1}$ and this runaway's is much higher. As noted by Evans \& Massey (2015), only two Galactic O stars are known to have peculiar velocities > $100 \mathrm{~km} \mathrm{~s}^{-1}$, and all three runaway RSGs in the Milky Way have peculiar velocities $<70 \mathrm{~km}$ $\mathrm{s}^{-1}$. We believe that the high peculiar velocity of this YSG points to it originating due to a supernova explosion following the original suggestion of Zwicky (1957) and Blaauw (1956a, 1956b).

Many runaway stars are found using their bow shocks, or a presence of an infrared excess (Gvaramadze et al. 2010). We estimated that in this case the bow shock would be around 3.0" away from the star. We first looked for any sign of the bow shock in MIPS SAGE-SMC data (Gordon et al. 2011) but sadly the resolution was too poor. We then obtained $\mathrm{H} \alpha$ imaging of the star using the Slope 1-m telescope at Las Campanas but again we saw nothing. Finally, we looked at MCELS data (Smith et al. 2000) to no avail. If there is a bow shock for this runaway star, we were not able to find it.

Another interesting point to make is that these runaway stars can act as dispersing mechanism for enriched elements; for example, this YSG will eventually explode as a supernova far away from where it was created. Since such a large fraction of OB stars are runaways, this must occur on a fairly regular basis. Thus, the process of runaway stars acts as a mechanism for dispersing elements throughout the galaxy. Although massive stars are well known to be the major source of many of the heavier elements (Maeder 1981), runaways 
have been largely ignored in favor of diffusion and similar processes as a means of dispersal (see, e.g., Pipino \& Matteucci 2009). Recent evidence, however, suggests that our own Solar System may have been created by a runaway Wolf-Rayet star (Tatischeff \& Sereville 2010)!

As future work, we hope to look into other potential YSG runaways that we found as part of a similar project in the Large Magellanic Cloud (LMC). One star in particular, J04530398-6937285 has a radial velocity of $373 \mathrm{~km} \mathrm{~s}^{-1}$, much higher than expected, and warrants further investigation 4 .

We would like to thank Maria Drout for help collecting the discovery spectra for this star as part of the larger project to identify all YSGs in the SMC. We would also like to thank Sean Points from the MCELS team who helped us try and find evidence of a bow shock. We very much appreciate the continued support of our work from the Arizona Time Allocation Committee. Finally, we would like to acknowledge the NSF grant AST-1612874.

\section{REFERENCES}

Ardeberg, A. \& Maurice, E. 1977, A\&AS, 30, 261

Azzopardi, M. \& Vigneau, J. 1979, A\&AS, 35, 353

Azzopardi, M., Vigneau, J. \& Macquet, M. 1975, A\&AS, 22, 285

Böhm-Vitense, E. 1981, ARAA 19, 295

Bouchet, P., Lequeux, J., Maurice, E., Prevot, L. \& Prevot-Burnichon, M. L. 1985, A\&A, 149,330

Blaauw, A. 1956a, PASP, 68, 495

Blaauw, A. 1956b, ApJ, 123, 408

Cannon, A. J. \& Pickering, E. C. 1918, Annals of Harvard College Observ., 91, 1

Carney, B. W., Janes, K. A. \& Flower, P. J. 1985, AJ, 90, 1196

Capuzzo-Dolcetta, R., \& Fragione, G. 2015, MNRAS, 454, 2677

Castelli, F., \& Kurucz, R. L. 2004, in Modeling of Stellar Atmospheres, IAU Symp. 210, ed. N. E. Piskunov, W.

\footnotetext{
${ }^{4}$ Another star, J04482407-7104012 (CPD-71²285, HD 268700) has a radial velocity of $401 \mathrm{~km} \mathrm{~s}^{-1}$, but Gaia's result results give a parallax measurement consistent with this star being a foreground halo giant at a distance of $350 \mathrm{pc}$.
} 
Cox, A. N. 2000, Allen's Astrophysical Quantities, 4th Edition (New York: Springer)

Cox, N. L. J. et al. 2012, A\&A, 537, A35

Ekström, s., et al., 2012, A\&A, 537, 146

Evans, C. J., Howarth, I. D., Irwin, M. J., Burnley, A. W. \& Harries, T. J. 2004, MNRAS, 353,601

Evans, K. A., \& Massey, P. 2015, AJ, 150, 149

Fehrenbach, C., \& Duflot, M. 1982, A\&AS, 48, 409

Florsch, A. 1972, Publ. Obs. Astron. Strausbourg, 2, 1

Fragione, G., \& Capuzzo-Dolcetta, R. 2016, MNRAS, 458, 2596

Georgy, C., Ekström, S., Granada, A., Meynet, G., Mowlavi, N., Eggenberger, P., \& Maeder, A., 2013, A\&A, 553, 24

Gies, D. R., \& Bolton, C. T. 1986, ApJS, 61, 419

Gonzàlez-Fernàdez, C., Dorda, R., Negueruela, I., \& Marco, A., 2015, A\&A, 578, 3

Gordon, K. D., et al. 2011, AJ, 142, 102

Gvaramadze, V. V., Kroupa, P., \& Pflamm-Altenburg, J., 2010, A\&A, 519, 33

Gvaramadze, V. V., Menten, K. M., \& Kniazev, A. Y. et al. 2014, MNRAS, 437, 843

Houk, N. \& Cowley, A. P. 1975, Univ. of Mich. Cat. of 2D Spect. Types for HD Stars, C01, 0

Humphreys, R. M. 1983, ApJ, 265, 176

Humphreys, R. M., \& McElroy, D. B. 1984, ApJ, 284, 565

Humphreys, R. M., Kudritzki, R. P. \& Groth, H. G. 1991, A\&A, 245, 593

Kaler, J. B. 2011, Stars and Their Spectra (Cambridge: Cambridge University Press), 186

Keenan, P. C., \& McNeil, R. C. 1989, ApJS, 71, 245

Kordopatis, G., Gilmore, G., Steinmetz, M. et al. 2013, AJ, 146, 134

Lennon, D. J. 1997, A\&A, 317, 871

Leonard, P. J. T., \& Duncan, M. J. 1990, AJ, 99, 608

Levesque, E. M., Massey, P., \& Olsen, K. A. G. 2006, ApJ, 645, 1102

Lü, P. K. 1971, Trans. of the Astron. Obs. Yale Univ., 31, 1

Maeder, A. 1981, A\&A, 101, 385

Massey, P. \& Olsen, K. A. G. 2003, AJ, 126, 2867 
Massey, P., Olsen, K. A. G., Hodge, P. W., Jacoby, G. H., McNeill, R. T., Smith, R. C., \& Strong, S. B. 2007, AJ, 133, 2393

Neugent, K. F., Massey, P., Skiff, B., Drout, M. R., Meynet, G., \& Olsen, K. A. G. 2010, ApJ, 719, 1784

Noriega-Crespo, A., van Buren, D., Cao, Y. and Dgani, R. 1997, AJ, 114, 837

Pipino, A., \& Matteucci, F. 2009, in The Age of Stars, IAU Symp. 258, ed. E. E. Mamajek, D. R. Soderblom, \& R. F. G. Wyse, (Cambridge: Cambridge Univ. Press), 39

Richter, O. -G., Tammann G. A. \& Huchtmeier, W. K. 1987, A\&A, 171, 33

Sanduleak, N. 1969, AJ, 74, 877

Sanduleak, N. 1989, AJ, 98, 925

Schlegel, D. J., Finkbeiner, D. P., \& Davis, M. 1998, ApJ, 500, 525

Smith, C., Leiton, R., \& Pizarro, S. 2000, ASPC, 221, 83

Stanimirović, S., Staveley-Smith, L. \& Jones, P. A. 2004, ApJ, 604, 176

Tatischeff, V., Duprat, J., \& de Sereville, N. 2010, ApJ, 714L, 26

Tonry, J. \& Davis, M. 1979, AJ, 84, 1511

van den Bergh, S. 2000, in The Galaxies of the Local Group (Cambridge: Cambridge University Press)

Wallerstein, G. 1984, AJ, 89, 1705

Zaritsky, D., Harris, J., Thompson, I. B., Grebel, E. K. \& Massey, P. 2002, AJ, 123, 855

Zwicky, F. 1957, Morphological Astronomy, (Berlin: Springer), 258 


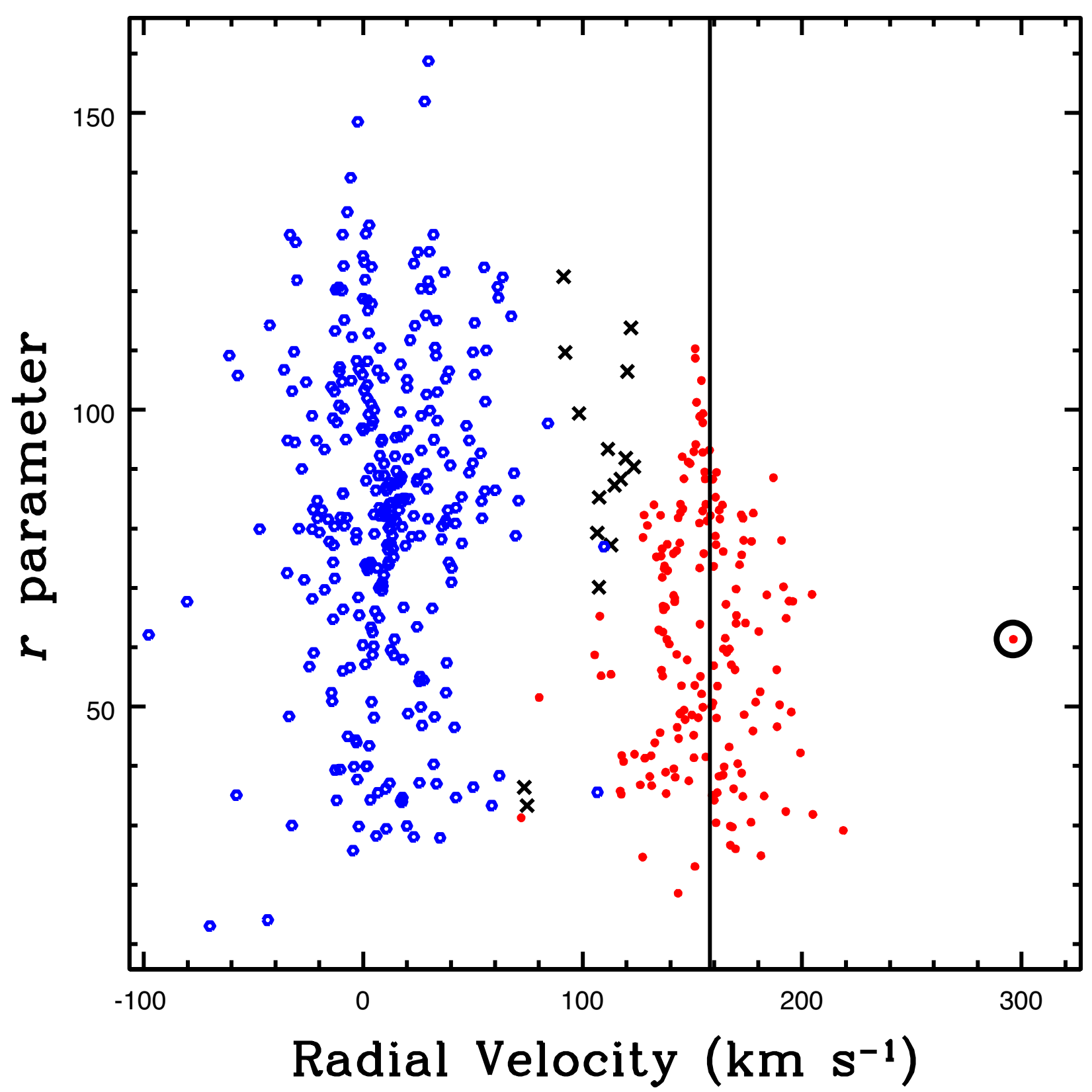

Fig. 1.- Separation of Foreground and SMC Yellow Stars. Blue open hexagons represent foreground yellow dwarfs. Notice how they're clustered around $0 \mathrm{~km} \mathrm{~s}^{-1}$. Red filled circles represent SMC yellow supergiants as they're clustered around the SMC's average heliocentric radial velocity of $158 \mathrm{~km} \mathrm{~s}^{-1}$. Black $\times \mathrm{s}$ in the middle represent stars with uncertain classifications. The y axis is the Tonry and Davis (1979) $r$ parameter which is a proxy for the error on each measurement with high values indicating lower errors. This paper discusses the star with the abnormally large radial velocity located at $\sim 300 \mathrm{~km} \mathrm{~s}^{-1}$. Data from Neugent et al. (2010) with a small revision as described in the text. 


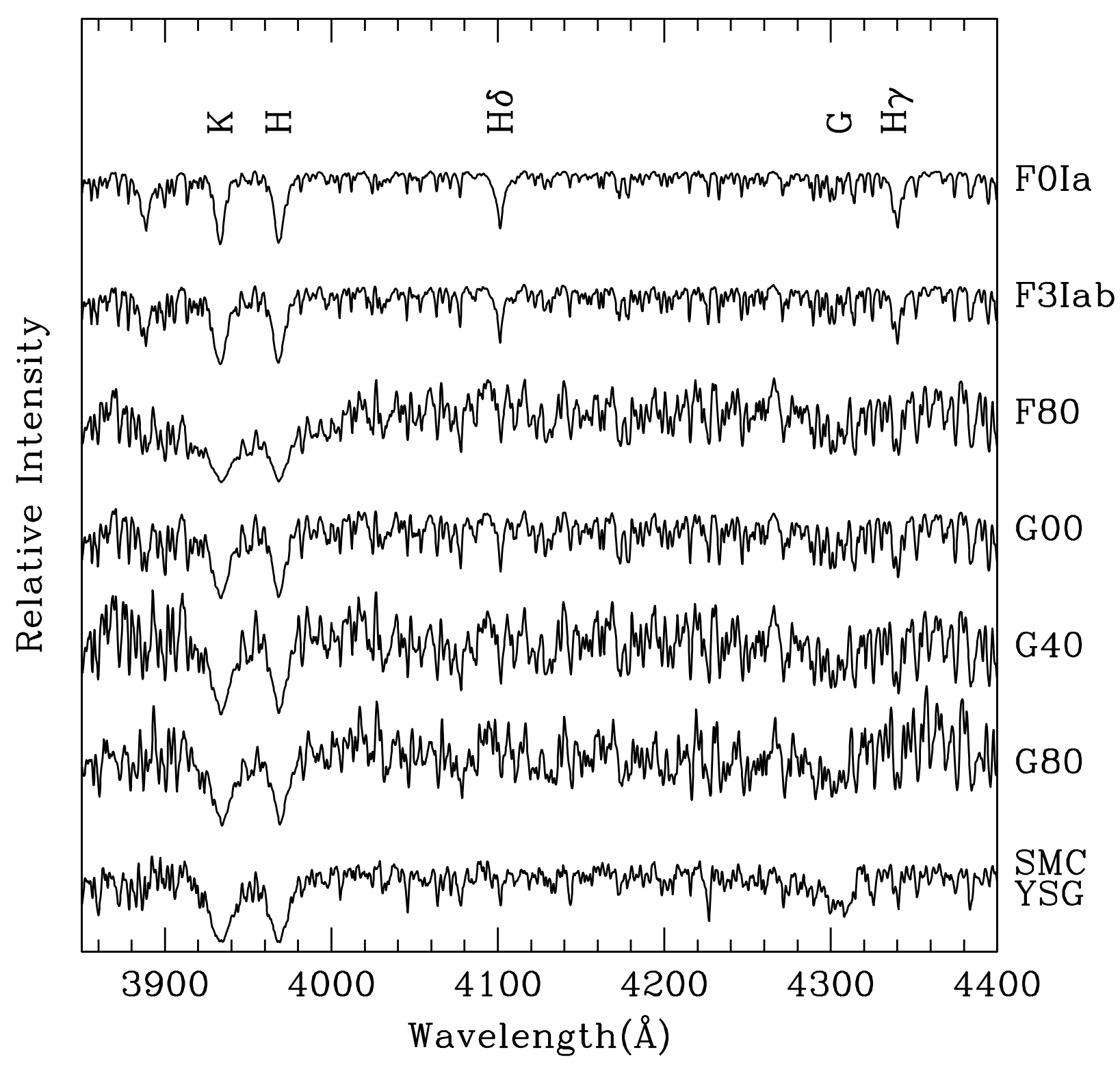

Fig. 2.- Spectral classification stars and runaway star spectrum. We classified the runaway YSG by comparing it to other LMC and SMC YSGs with well-established types. The prominent G-band, abundance of metal lines and lack of hydrogen lines point to it being a G5-8 I. The spectrum of the YSG is shown on the bottom. The stars shown are the spectral standards Sk 105 (F0 Ia), Sk 55 (F3 Iab), HD 271182 (F8 0), HD 269953 (G0 0), HD 269723 (G4 0), HD 268757 (G8 0), along with our YSG runaway, J01020100-7122208. We are making these data available electronically as the "Data behind the Figure." 


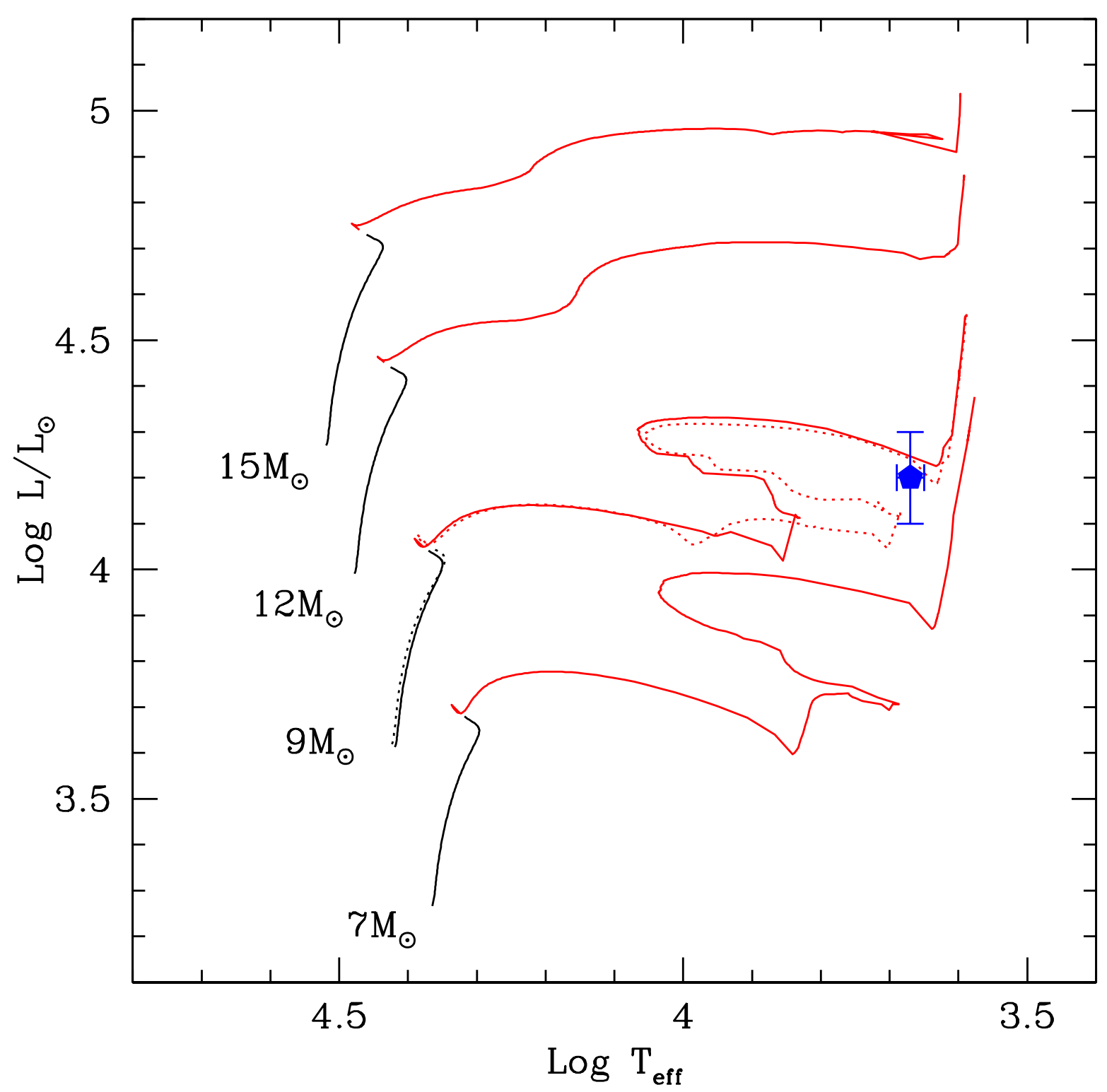

Fig. 3.- H-R diagram along with Geneva evolution tracks. The luminosity and temperature are shown with errors. The Geneva $\mathrm{z}=0.006$ evolution tracks are plotted in black during the main-sequence phase and then red during the evolved stages. These tracks were interpolated to a metallicity of $\mathrm{z}=0.004$ (SMC-like) using the interactive web tool at the Geneva web site (https://obswww . unige.ch/Recherche/evoldb/index/Interpolation/) starting with the B-type stars grid published by Georgy et al. (2013) and adopting an initial equatorial rotation velocity of $40 \%$ of the breakup speed $\left(\Omega / \Omega_{\text {crit }}=0.568\right)$. The dotted $9 M_{\odot}$ track is for a value of $\Omega / \Omega_{\text {crit }}=0.379$. Regardless of the assumption, the tracks indicate a mass of $9 M_{\odot}$ and an age of about $30 \mathrm{Myr}$ for the YSG runaway. 


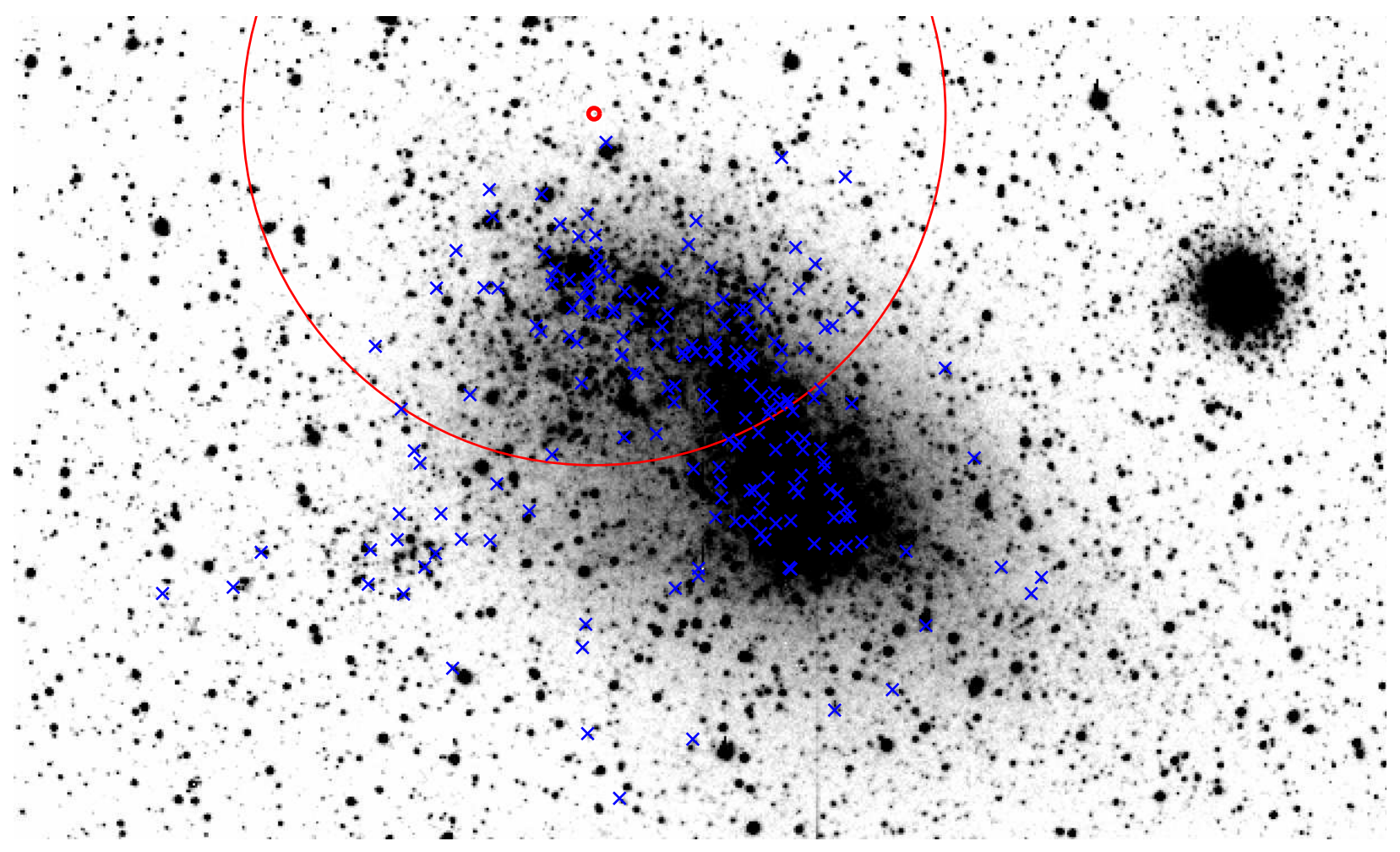

Fig. 4.- Location of the runaway YSG within the SMC. The location is indicated by the small red circle while the location of the other SMC YSGs are shown as blue $\times$ s. The large red circle denotes the $1.6^{\circ}$ the star would travel over 10 million years if its transverse velocity is similar to its radial peculiar velocity. 
Table 1. Observations

\begin{tabular}{|c|c|c|c|c|c|c|}
\hline Date & HJD & Telescope/Instrument & Wavelength & Resolution & Exp. time (s) & $\operatorname{HRV}\left(\mathrm{km} \mathrm{s}^{-1}\right)$ \\
\hline 09 Oct 2009 & 2455113.67 & CTIO 4-m/Hydra & $7300-9050 \AA$ & 3000 & 900 & 296.4 \\
\hline 16 Aug 2017 & 2457981.87 & DuPont $2.5-\mathrm{m} /$ Echelle & $3500-9050 \AA *$ & 45000 & 1800 & 298.0 \\
\hline 31 Dec 2017 & 2458118.59 & Baade 6.5/MagE & $3200 \AA-1 \mu \mathrm{m}$ & 4100 & 1800 & 305.1 \\
\hline 1 Jan 2018 & 2458119.58 & Baade 6.5/MagE & $3200 \AA-1 \mu \mathrm{m}$ & 4100 & 600 & 305.7 \\
\hline
\end{tabular}

${ }^{*}$ Underexposed below $4200 \AA$.

Table 2. Radial Velocities of Classification Standards

\begin{tabular}{lllllr}
\hline & Type & MagE* & Published* & Ref & (MagE - Published)* \\
\hline Sk 55 & F3 Iab & 168.4 & 159.9 & 1 & 8.5 \\
HD271182 & F8 0 & 309.9 & 323.9 & 1 & -14.0 \\
HD269953 & G0 0 & 259.0 & 240.4 & 1 & 18.6 \\
HD269723 & G4 0 & 323.4 & 332.0 & 2 & -8.6 \\
HDE268757 & G8 0 & 284.8 & 271.6 & 1 & 13.2 \\
\hline
\end{tabular}

*units of $\mathrm{km} \mathrm{s}^{-1}$

${ }^{1}$ Kordopatis et al. 2013

${ }^{2}$ Fehrenbach \& Duflot 1982 
Table 3. Properties of Observed Targets

\begin{tabular}{|c|c|c|c|c|c|c|c|c|c|c|c|c|c|c|c|}
\hline 2MASS & $\alpha(\mathrm{J} 2000)$ & $\delta(\mathrm{J} 2000)$ & K & $J-K$ & $\bar{V}$ & $B-V$ & Ref. $^{b}$ & $\begin{array}{c}\mathrm{Vel} \\
\left(\mathrm{km} \mathrm{s}^{-1}\right)\end{array}$ & $r^{\mathrm{c}}$ & Category $^{\mathrm{d}}$ & $\begin{array}{c}\text { Hydra } \\
\text { Class }\end{array}$ & $\begin{array}{c}\text { Literature } \\
\text { Class }\end{array}$ & Ref. $^{\text {e }}$ & Comments $^{\mathrm{f}}$ & \\
\hline J00342570-7322334 & 03425.69 & -732233.5 & 11.6 & 0.25 & 12.79 & $\ldots$ & 3 & -9.98 & 100.2 & 3 & & 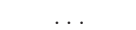 & $\ldots$ & & \\
\hline J00342883-7328192 & 03428.82 & $\begin{array}{llll}-73 & 28 & 19.3\end{array}$ & 10.72 & 0.59 & 13.03 & 0.97 & 4 & 91.12 & 109.7 & 2 & $\ldots$ & G6IV & 12 & $\ldots$ & \\
\hline J00344094-7319366 & 03440.94 & -731936.7 & 9.18 & 0.69 & 11.73 & $\ldots$ & 3 & 38.02 & 106.5 & 3 & $\ldots$ & $\ldots$ & $\ldots$ & $\ldots$ & \\
\hline J00345529-7213044 & 03455.30 & $\begin{array}{lll}-72 & 13 & 04.4\end{array}$ & 11.58 & 0.31 & 12.93 & $\ldots$ & 3 & -10.29 & 56 & 3 & $\ldots$ & & $\ldots$ & & \\
\hline J00345992-7339156 & 03459.91 & -733915.8 & 10.66 & 0.33 & 12.13 & $\ldots$ & 3 & 9.92 & 77.3 & 3 & $\ldots$ & F8 & 6 & Flo 72 & \\
\hline J00350326-7332586 & 03503.25 & -733258.8 & 10.77 & 0.8 & 13.8 & 1.54 & 4 & 134.82 & 75.4 & 1 & $\ldots$ & G6Ib/II & 12 & SkKM 2 & \\
\hline J00351576-7340108 & 03515.75 & $\begin{array}{lll}-73 & 40 & 10.9\end{array}$ & 10.25 & 0.47 & 12.13 & 0.85 & 4 & -9.58 & 115.1 & 3 & $\ldots$ & $\mathrm{K} 2 \mathrm{~V}$ & 12 & ... & \\
\hline J00353473-7341121 & 03534.74 & $-7341 \quad 12.3$ & 11.04 & 0.37 & 12.55 & $\ldots$ & 3 & -16.38 & 77.8 & 3 & $\ldots$ & $\ldots$ & $\ldots$ & $\ldots$ & \\
\hline J00353564-7205134 & 03535.64 & $-7205 \quad 13.5$ & 11.28 & 0.34 & 12.7 & & 3 & 2.51 & 63.3 & 3 & & & & & \\
\hline J00354135-7210010 & 03541.38 & $-72 \quad 1001.0$ & 11.51 & 0.31 & 12.79 & $\ldots$ & 3 & 18.31 & 77.1 & 3 & $\ldots$ & $\ldots$ & $\ldots$ & $\ldots$ & \\
\hline J00361882-7216201 & 03618.86 & -721620.1 & 10.54 & 0.77 & 13.38 & 1.16 & 4 & $\begin{array}{r}13.01 \\
-13.89\end{array}$ & 71.6 & 3 & $\ldots$ & $\ldots$ & $\ldots$ & $\ldots$ & \\
\hline J00362691-7230502 & 03626.94 & -723050.2 & 10.34 & 0.33 & 11.69 & $\ldots$ & 3 & -12.99 & 97.8 & 3 & $\ldots$ & $\mathrm{F} 7$ & 6 & Flo 83 & \\
\hline J00364059-7319091 & 03640.59 & $\begin{array}{llll}-73 & 19 & 09.2\end{array}$ & 10.06 & 0.6 & 12.3 & & 3 & 15.92 & 107.7 & 3 & $\ldots$ & KoIV & 12 & & \\
\hline J00365948-7338435 & 03659.49 & -733843.5 & 10.99 & 0.45 & 12.83 & 0.82 & 4 & 14.72 & 87.6 & 3 & $\ldots$ & & $\ldots$ & & \\
\hline J00370536-7326159 & 03705.36 & -732616.0 & 10.46 & 0.78 & 13.51 & 1.55 & 4 & 122.32 & 90.4 & 2 & $\ldots$ & $\ldots$ & $\ldots$ & SkKM 6 & \\
\hline J00371053-7349353 & 03710.53 & -734935.4 & 11.22 & 0.06 & 11.53 & $\ldots$ & 3 & -44.48 & 14 & 3 & & A2 & 1 & HD 3542 & \\
\hline J00375257-7201070 & 03752.59 & $\begin{array}{llll}-72 & 01 & 07.2\end{array}$ & 11.69 & 0.35 & 13.24 & 0.59 & 4 & 19.41 & 91.7 & 3 & & $\cdots$ & $\ldots$ & $\ldots$ & \\
\hline J00381017-7311321 & 03810.16 & -731132.2 & 8.98 & 0.3 & 10.21 & $\ldots$ & 3 & 6.82 & 82.1 & 3 & & Fo & 9 & HD 3659; Flo 96 & \\
\hline J00382510-7400464 & 03825.08 & -740046.5 & 11.22 & 0.38 & 12.86 & $\ldots$ & 3 & -33.43 & 30 & 3 & $\ldots$ & & 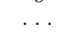 & & \\
\hline J00382659-7359394 & 03826.57 & -735939.6 & 6.23 & 0.92 & 9.49 & $\ldots$ & 3 & -10.03 & 85.8 & 3 & $\ldots$ & K5 & 5 & CPD-74 54 & \\
\hline J00383391-7409574 & 03833.91 & -740957.4 & 9.97 & 0.67 & 12.58 & $\ldots$ & 3 & 1.77 & 43.4 & 3 & $\ldots$ & $\ldots$ & $\ldots$ & ... & • \\
\hline J00390248-7357293 & 03902.47 & $-73 \quad 5729.3$ & 11.32 & 0.3 & 12.56 & 0.65 & 4 & -3.93 & 44 & 3 & $\ldots$ & $\ldots$ & $\ldots$ & $\ldots$ & a. \\
\hline J00390455-7332038 & 03904.56 & -733203.8 & 10.77 & 0.33 & 12.36 & $\ldots$ & 3 & -21.78 & 81.7 & 3 & $\ldots$ & $\ldots$ & $\ldots$ & & I \\
\hline J00391218-7256366 & 03912.18 & -725636.7 & 9.52 & 0.89 & 13.03 & 1.56 & 2 & 130.28 & 41.7 & 1 & $\ldots$ & $\ldots$ & $\ldots$ & SkKM 8 & \\
\hline J00391298-7222186 & $\begin{array}{l}03912.99 \\
0\end{array}$ & -722218.8 & 11.14 & 0.36 & 12.73 & 0.8 & 5 & 47.31 & 94.8 & 3 & $\ldots$ & $\ldots$ & $\ldots$ & ... & \\
\hline J00391532-7237084 & 03915.33 & $-72 \quad 3708.4$ & 11.31 & 0.41 & 13.02 & $\ldots$ & 3 & 7.68 & 69.5 & 3 & & & & & \\
\hline J00391534-7219542 & 03915.35 & -721954.4 & 11.04 & 0.37 & 12.53 & 0.39 & 4 & 13.51 & 92.2 & 3 & & $\ldots$ & $\ldots$ & & \\
\hline J00391791-7301226 & 03917.92 & -730122.6 & 9.24 & 0.52 & 11.16 & $\ldots$ & 3 & 29.28 & 126.6 & 3 & $\ldots$ & G7V & 12 & $\ldots$ & \\
\hline J00393161-7233027 & 03931.60 & -723302.8 & 10.4 & 0.48 & 12.44 & 0.77 & 4 & $\begin{array}{l}-14.79 \\
-7.20\end{array}$ & 98.6 & 3 & $\ldots$ & $\ldots$ & $\ldots$ & $\ldots$ & \\
\hline J00393530-7157347 & 03935.32 & -715734.8 & 10.97 & 0.28 & 12.28 & $\ldots$ & 3 & -4.19 & 78.2 & 3 & $\ldots$ & $\ldots$ & $\ldots$ & $\ldots$ & \\
\hline J00393667-7406537 & 03936.66 & $-7406 \quad 53.7$ & 11.37 & 0.46 & 13.27 & $\ldots$ & 3 & 16.97 & 34.7 & 3 & $\ldots$ & & $\ldots$ & $\ldots$ & \\
\hline J00400920-7234423 & $040 \quad 09.21$ & -723442.5 & 10.86 & 0.21 & 11.85 & $\ldots$ & 3 & -3.15 & 68.4 & 3 & $\ldots$ & F0 & 6 & Flo 110 & \\
\hline J00400936-7305417 & $040 \quad 09.35$ & $\begin{array}{lll}-73 & 05 & 41.8\end{array}$ & 10.05 & 0.62 & 12.46 & $\ldots$ & 3 & 25.38 & 49.9 & 3 & $\ldots$ & K1IV & 12 & & \\
\hline J00401981-7334106 & 04019.80 & -733410.5 & 10.94 & 0.32 & 12.41 & 0.58 & 1 & 15.02 & 88 & 3 & & G0III/IV & 22 & Flo 111 & \\
\hline J00405279-7415045 & 04052.80 & $-74 \quad 1504.5$ & 10.73 & 0.61 & 13.09 & $\ldots$ & 3 & -34.73 & 48.4 & 3 & $\ldots$ & G8III/IV & 22 & $\ldots$ & \\
\hline J00410308-7407593 & 04103.07 & -740759.4 & 10.8 & 0.41 & 12.39 & 0.69 & 4 & -27.03 & 104.7 & 3 & & ... & $\ldots$ & & \\
\hline J00410383-7200377 & $\begin{array}{lll}0 & 41 & 03.82\end{array}$ & -720038.0 & 10.62 & 0.28 & 11.91 & $\ldots$ & 3 & 25.41 & 120.4 & 3 & $\ldots$ & F7V & 6 & Flo 117 & \\
\hline J00411604-7232167 & 04116.05 & $-72 \quad 32 \quad 16.8$ & 8.66 & 0.8 & 11.63 & $\cdots$ & 3 & 203.58 & 68.9 & 1 & $\cdots$ & G6Iab & 12 & $\cdots$ & \\
\hline J00413588-7410375 & 04135.88 & -741037.5 & 6.47 & 0.69 & 9.01 & $\ldots$ & 3 & -8.13 & 133.3 & 3 & $\ldots$ & K1III & 9 & HD 4067 & \\
\hline J00414144-7334317 & 04141.47 & -733431.7 & 8.09 & 0.84 & 11.17 & 1.32 & 1 & -10.48 & 120.1 & 3 & $\ldots$ & & $\cdots$ & & \\
\hline J00414223-7216541 & 04142.25 & -721654.2 & 11.22 & 0.35 & 12.75 & & 3 & -10.59 & 104.7 & 3 & $\ldots$ & & $\ldots$ & & \\
\hline J00414344-7343239 & 04143.45 & $\begin{array}{l}-734323.9 \\
\end{array}$ & 9.49 & 0.58 & 12.09 & 1.33 & 1 & 97.42 & 99.4 & 2 & $\ldots$ & G0Iab & 12 & HV 821; RMC 1 & \\
\hline J00414355-7329119 & 04143.57 & -732911.9 & 9.04 & 0.64 & 11.45 & 1.04 & 1 & 46.02 & 97.3 & 3 & & K2IV & 12 & ... & \\
\hline J00420472-7203063 & $\begin{array}{lll}0 & 42 & 04.72\end{array}$ & $\begin{array}{lll}-72 & 03 & 06.5\end{array}$ & 10.49 & 0.28 & 11.7 & $\ldots$ & 3 & 21.11 & 78.6 & 3 & & F5I: & 6 & Flo 131 & \\
\hline J00423897-7355507 & 04238.96 & -735550.6 & 11.25 & 0.32 & 12.59 & 0.57 & 1 & 10.67 & 80.2 & 3 & $\ldots$ & $\ldots$ & $\ldots$ & $\ldots$ & \\
\hline J00424953-7338083 & 04249.54 & -733808.2 & 10.21 & 0.56 & 12.46 & 0.88 & 1 & -6.68 & 139.1 & 3 & $\ldots$ & G8IV & 12 & $\ldots$ & \\
\hline J00430535-7328062 & 04305.36 & -732806.1 & 11.11 & 0.58 & 13.3 & 0.87 & 1 & $\begin{array}{l}-0.00 \\
-2.76\end{array}$ & $\begin{array}{r}19.1 \\
29.8\end{array}$ & 3 & $\ldots$ & & $\ldots$ & $\ldots$ & \\
\hline J00430758-7327500 & 04307.57 & -732749.8 & 11.04 & 0.34 & 12.52 & 0.61 & 1 & 17.62 & 85.1 & 3 & $\ldots$ & & 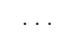 & & \\
\hline J00431164-7323109 a & $043 \quad 11.64$ & $\begin{array}{lll}-73 & 23 & 10.8\end{array}$ & 11.5 & 0.18 & 11.98 & 0.04 & 2 & 79.33 & 51.5 & 1 & $\ldots$ & B8Ia+ & 7 & $\mathrm{Sk} 3 ; \mathrm{AzV} 2 ; \mathrm{RMC} 2$ & \\
\hline J00431458-7330139 & 04314.58 & -733013.8 & 11.19 & 0.31 & 12.58 & 0.52 & 1 & 68.63 & 78.8 & 3 & $\ldots$ & & $\ldots$ & & \\
\hline
\end{tabular}


Table 3-Continued

\begin{tabular}{|c|c|c|c|c|c|c|c|c|c|c|c|c|c|c|c|}
\hline 2MASS & $\alpha(\mathrm{J} 2000)$ & $\delta(\mathrm{J} 2000)$ & $K$ & $J-K$ & V & $B-V$ & Ref. $^{b}$ & $\begin{array}{c}\mathrm{Vel} \\
\left(\mathrm{km} \mathrm{s}^{-1}\right)\end{array}$ & $r^{\mathrm{c}}$ & Category $^{\mathrm{d}}$ & $\begin{array}{c}\text { Hydra } \\
\text { Class }\end{array}$ & $\begin{array}{c}\text { Literature } \\
\text { Class }\end{array}$ & Ref. $^{\mathrm{e}}$ & Comments $^{\mathrm{f}}$ & \\
\hline J00431497-7337064 & 04314.96 & -733706.3 & 9.34 & 0.68 & 11.85 & 1.06 & 1 & -15.48 & 103.9 & 3 & & K1III & 12 & Flo 146 & \\
\hline J00431668-7400527 & 04316.66 & -740052.5 & 11.01 & 0.41 & 12.72 & 0.76 & 1 & 53.27 & 81.7 & 3 & $\ldots$ & $\ldots$ & $\ldots$ & $\ldots$ & \\
\hline J00431939-7209434 & 04319.39 & -720943.5 & 11.46 & 0.41 & 13.25 & & 3 & 10.83 & 74.4 & 3 & G8V & $\ldots$ & $\ldots$ & $\ldots$ & \\
\hline J00433304-7137483 & 04333.04 & -713748.3 & 11.78 & 0.29 & 13.21 & $\cdots$ & 3 & -12.83 & 34.2 & 3 & $\mathrm{G} 2 \mathrm{~V}$ & $\cdots$ & $\cdots$ & $\cdots$ & \\
\hline J00433619-7217166 & 04336.20 & $-7217 \quad 16.6$ & 11.11 & 0.58 & 13.36 & 1 & 4 & -23.17 & 59.1 & 3 & G8I & $\ldots$ & $\ldots$ & $\ldots$ & \\
\hline J00434441-7401260 & 04344.39 & -740125.9 & 10.56 & 0.91 & 14.01 & 1.42 & 1 & 135.57 & 62.6 & 1 & & $\ldots$ & $\ldots$ & $\ldots$ & \\
\hline J00434661-7133027 & 04346.60 & -713302.7 & 10.26 & 0.7 & 12.93 & $\ldots$ & 3 & -35.33 & 72.5 & 3 & $\ldots$ & $\ldots$ & $\ldots$ & $\ldots$ & \\
\hline J00440978-7325464 & 04409.78 & -732546.5 & 9.83 & 0.68 & 12.5 & 1.18 & 1 & 49.24 & 91 & 3 & $\ldots$ & $\mathrm{K} 2.5 \mathrm{IV}$ & 12 & $\ldots$ & \\
\hline J00441154-7211122 & 04411.55 & $-72 \quad 11 \quad 12.3$ & 10.52 & 0.72 & 13.21 & $\ldots$ & 3 & 70.23 & 84.7 & 3 & G8III & $\ldots$ & $\ldots$ & $\ldots$ & \\
\hline J00442211-7134588 & 04422.11 & -713458.7 & 11.58 & 0.18 & 12.53 & $\ldots$ & 3 & 30.77 & 66.6 & 3 & & $\ldots$ & & $\ldots$ & \\
\hline J00442921-7214555 & 04429.21 & $-72 \quad 1455.4$ & 8.77 & 0.26 & 9.95 & $\ldots$ & 3 & 1.13 & 74.1 & 3 & F5II & F3V & 9 & HD 4344; Flo 159 & \\
\hline J00443797-7342507 & 04437.96 & -734250.8 & 11.15 & 0.56 & 13.39 & 1 & 1 & 34.07 & 27.9 & 3 & & $\ldots$ & $\ldots$ & $\ldots$ & \\
\hline J00444592-7154502 & 04445.92 & -715450.2 & 11.46 & 0.42 & 13.14 & $\ldots$ & 3 & -14.77 & 50.9 & 3 & G8V & $\ldots$ & $\ldots$ & $\ldots$ & \\
\hline J00450014-7313452 & 04500.16 & $-73 \quad 1345.2$ & 6.35 & 0.91 & 9.58 & $\ldots$ & 3 & 12.04 & 82.3 & 3 & & Ko & 1 & HD 4420 & \\
\hline J00450023-7222303 & 04500.26 & -722230.3 & 11.52 & 0.38 & 13.1 & 0.56 & 4 & -11.07 & 39.4 & 3 & G2I & $\cdots$ & & & \\
\hline J00450023-7310065 & $045 \quad 00.24$ & -731006.5 & 10.52 & 0.63 & 12.97 & 1.03 & 1 & 40.84 & 46.5 & 3 & & KOII/III & 22 & $\mathrm{AzV} 23 \mathrm{~F}$ & \\
\hline J00451179-7307093 & 04511.80 & $\begin{array}{llll}-73 & 07 & 09.2\end{array}$ & 9.16 & 0.7 & 11.71 & 1.03 & 1 & -21.74 & 84.7 & 3 & $\ldots$ & G3II/III & 22 & $\ldots$ & \\
\hline J00451547-7415010 & $045 \quad 15.48$ & $\begin{array}{llll}-74 & 15 & 00.9\end{array}$ & 11.67 & 0.31 & 13.16 & 0.59 & 5 & -11.93 & 100.8 & 3 & & $\ldots$ & $\ldots$ & $\ldots$ & \\
\hline J00451741-7415422 & $045 \quad 17.40$ & $-74 \quad 15 \quad 42.1$ & 11.1 & 0.61 & 13.42 & 0.89 & 4 & 31.37 & 95 & 3 & & $\ldots$ & $\ldots$ & $\ldots$ & \\
\hline J00451949-7144466 & 04519.50 & -714446.6 & 10.68 & 0.65 & 13.08 & $\ldots$ & 3 & -5.83 & 112.3 & 3 & $\ldots$ & $\ldots$ & $\ldots$ & $\ldots$ & I \\
\hline J00452017-7202576 & $045 \quad 20.17$ & -720257.6 & 11.67 & 0.24 & 12.86 & $\ldots$ & 3 & 1.53 & 73 & 3 & F5V & $\ldots$ & $\ldots$ & $\ldots$ & $\mapsto$ \\
\hline J00454118-7156104 & 04541.19 & $\begin{array}{lll}-71 & 56 & 10.5\end{array}$ & 10.96 & 0.7 & 13.53 & 1.07 & 4 & 13.73 & 61.3 & 3 & G8V & $\ldots$ & $\ldots$ & $\ldots$ & $-v$ \\
\hline J00454793-7144342 & 04547.93 & -714434.2 & 11.35 & 0.33 & 12.77 & $\ldots$ & 3 & -3.73 & 108.3 & 3 & $\cdots$ & $\ldots$ & $\ldots$ & $\ldots$ & | \\
\hline J00460240-7321000 & 04602.39 & -732100.0 & 10.86 & 0.75 & 13.77 & 1.37 & 1 & 133.94 & 62.9 & 1 & & G5Iab & 12 & & \\
\hline J00462301-7146568 a & 04623.02 & -714656.7 & 6.07 & 0.86 & 9.19 & $\ldots$ & 3 & 1.15 & 101.9 & 3 & K2III & K2III & 9 & HD 4577 & \\
\hline J00462423-7320084 & 04624.23 & -732008.4 & 10.18 & 0.23 & 11.3 & $\cdots$ & 3 & -2.66 & 65.4 & 3 & & F9III & 12 & Flo 181 & \\
\hline J00462977-7158227 & 04629.77 & -715822.6 & 11.09 & 0.46 & 13.15 & 0.92 & 5 & -29.77 & 80 & 3 & KoIII & $\ldots$ & $\ldots$ & $\ldots$ & \\
\hline J00463746-7316142 a & 04637.48 & $\begin{array}{lll}-73 & 16 & 14.2\end{array}$ & 11.21 & 0.34 & 12.75 & 0.71 & 1 & 28.3 & 102.6 & 3 & $\mathrm{G} 2 \mathrm{~V}$ & $\ldots$ & $\ldots$ & $\ldots$ & \\
\hline J00464324-7126321 & 04643.25 & -712632.2 & 10.33 & 0.47 & 12.25 & $\ldots$ & 3 & 9.57 & 87 & 3 & $\ldots$ & $\ldots$ & $\ldots$ & $\ldots$ & \\
\hline J00464964-7120125 & 04649.65 & $\begin{array}{llll}-71 & 20 & 12.7\end{array}$ & 8.44 & 0.78 & 11.23 & $\ldots$ & 3 & 6.17 & 88.9 & 3 & $\ldots$ & $\ldots$ & $\ldots$ & $\ldots$ & \\
\hline J00464984-7313525 a & 04649.85 & -731352.5 & 10.21 & 0.91 & 13.58 & 1.19 & 1 & 106.8 & 70.1 & 2 & $\ldots$ & KOIab/Ib & 22 & $\ldots$ & \\
\hline J00465308-7242518 & 04653.11 & -724251.7 & 10.23 & 0.53 & 12.04 & 0.63 & 2 & 141.48 & 38.1 & 1 & $\cdots$ & GOIab & 12 & HV 824; cepheid & \\
\hline J00465795-7132158 & 04657.95 & $\begin{array}{llll}-71 & 32 & 15.9\end{array}$ & 11 & 0.42 & 12.67 & & 3 & -21.93 & 94.8 & 3 & & $\cdots$ & $\cdots$ & & \\
\hline J00465844-7216256 & 04658.44 & $-7216 \quad 25.7$ & 9.89 & 0.88 & 13.16 & 1.25 & 2 & 153.53 & 105 & 1 & F8 & $\ldots$ & & SkKM 15 & \\
\hline J00470092-7322137 a & 04700.92 & -732213.6 & 11.61 & 0.37 & 13.22 & 0.51 & 1 & 166.8 & 26.7 & 1 & A5:I: & $\ldots$ & $\ldots$ & $\ldots$ & \\
\hline J00470493-7249319 & 04704.93 & -724931.8 & 8.59 & 0.26 & 9.78 & 0.46 & 2 & -5.32 & 25.7 & 3 & $\ldots$ & F9IV/V & 12 & HD 4651 & \\
\hline J00470584-7311315 a & 04705.87 & -731131.6 & 10.06 & 0.84 & 13.2 & 1.31 & 1 & 135.7 & 71.8 & 1 & $\ldots$ & $\ldots$ & $\cdots$ & SkKM 18 & \\
\hline J00470689-7241144 & 04706.89 & $-7241 \quad 14.4$ & 8.57 & 0.71 & 11.45 & & 3 & 12.78 & 76.6 & 3 & $\ldots$ & K3III/IV & 22 & $\ldots$ & \\
\hline J00470869-7314119 a & 04708.69 & $\begin{array}{llll}-73 & 14 & 11.9\end{array}$ & 9.94 & 0.9 & 13 & 0.69 & 1 & 112.3 & 77.2 & 2 & $\cdots$ & G6Ia+Bne & 22 & LHA $115-\mathrm{S} 7$; RMC & $14 \mathrm{~F}$ \\
\hline J00471122-7130154 & 04711.22 & $\begin{array}{llll}-71 & 30 & 15.5\end{array}$ & 11.37 & 0.34 & 13.05 & 0.5 & 4 & 2.97 & 100.9 & 3 & $\ldots$ & $\ldots$ & $\ldots$ & $\ldots$ & \\
\hline J00471355-7208364 & 04713.55 & -720836.5 & 9.83 & 0.6 & 12.18 & & 3 & 66.93 & 115.8 & 3 & G8III & $\ldots$ & $\ldots$ & $\ldots$ & \\
\hline J00472498-7137576 & 04724.98 & -713757.6 & 11.13 & 0.48 & 13.01 & 0.62 & 5 & 36.87 & 81.4 & 3 & $\ldots$ & $\ldots$ & $\ldots$ & $\ldots$ & \\
\hline J00473213-7407291 & 04732.13 & $\begin{array}{lll}-74 & 07 & 29.2\end{array}$ & 12.61 & 0.02 & 12.97 & $\ldots$ & 3 & 126.47 & 24.7 & 1 & $\ldots$ & A2II & 3 & $2 \mathrm{dFS} 0655$ & \\
\hline J00473438-7140232 & 04734.37 & -714023.2 & 12.03 & 0.19 & 13.02 & $\ldots$ & 3 & 71.27 & 31.3 & 1 & $\ldots$ & $\ldots$ & $\cdots$ & $\ldots$ & \\
\hline J00473872-7307488 & 04738.74 & -730748.8 & 12.17 & 0.02 & 12.24 & -0.07 & 2 & 143.7 & 48.8 & 1 & B2I & B5Ia & 7 & $\mathrm{AzV} 22 ; \mathrm{Sk} 15$ & \\
\hline J00473889-7322539 a & 04738.90 & -732253.8 & 11.96 & 0.06 & 12.18 & 0.09 & 1 & 166.2 & 43.2 & 1 & $\mathrm{~B} 2 \mathrm{I}$ & B3Ia & 7 & $\mathrm{AzV} 23 ;$ Sk 17 & \\
\hline J00474278-7130575 & 04742.78 & -713057.6 & 10.05 & 0.59 & 12.28 & $\ldots$ & 3 & -30.93 & 121.8 & 3 & $\ldots$ & ... & $\ldots$ & ... & \\
\hline J00474745-7355289 & 04747.45 & -735529.0 & 8.47 & 0.6 & 10.66 & $\ldots$ & 3 & 4.07 & 99.9 & 3 & & $\ldots$ & $\ldots$ & $\ldots$ & \\
\hline J00474903-7218195 & 04749.04 & -721819.8 & 9.9 & 0.59 & 12.29 & 0.94 & 4 & 50.23 & 114.7 & 3 & KoIII & $\ldots$ & $\ldots$ & $\ldots$ & \\
\hline J00475033-7314227 & 04750.35 & -731422.7 & 11.63 & 0.09 & 12.14 & 0.14 & 1 & 112.46 & 55.5 & 1 & B8I & $\mathrm{A} 2 \mathrm{I}$ & 8 & $\mathrm{AzV} 27 ; \mathrm{Sk} 19$ & \\
\hline
\end{tabular}


Table 3-Continued

\begin{tabular}{|c|c|c|c|c|c|c|c|c|c|c|c|c|c|c|}
\hline 2MASS & $\alpha(\mathrm{J} 2000)$ & $\delta(\mathrm{J} 2000)$ & K & $J-K$ & V & $B-V$ & Ref. $^{\text {b }}$ & $\begin{array}{c}\mathrm{Vel} \\
\left(\mathrm{km} \mathrm{s}^{-1}\right)\end{array}$ & $r^{\mathrm{c}}$ & Category $^{\mathrm{d}}$ & $\begin{array}{c}\text { Hydra } \\
\text { Class }\end{array}$ & $\begin{array}{c}\text { Literature } \\
\text { Class }\end{array}$ & Ref. $^{e}$ & Comments $^{f}$ \\
\hline J00475150-7144545 & 04751.49 & -714454.5 & 11.16 & 0.37 & 12.71 & .. & 3 & -4.13 & 44.4 & 3 & & $\ldots$ & $\cdots$ & \\
\hline J00480629-7306379 a & 04806.31 & -730638.0 & 11.5 & 0.21 & 12.47 & 0.27 & 1 & 104.9 & 58.7 & 1 & B8:I: & A0 & 14 & $\mathrm{AzV} 31 ; \mathrm{Sk} 20$ \\
\hline J00480954-7221283 & $048 \quad 09.55$ & -722128.6 & 10.54 & 0.92 & 13.93 & 1.46 & 1 & 152.93 & 63.9 & 1 & $\ldots$ & G8Iab & 12 & SkKM 26 \\
\hline J00482593-7146264 & 04825.94 & -714626.3 & 11.02 & 0.57 & 13.31 & 0.98 & 5 & 33.17 & 98.2 & 3 & $\ldots$ & & $\cdots$ & $\ldots$ \\
\hline J00482632-7209508 & 04826.33 & -720950.9 & 10.89 & 0.33 & 12.38 & 0.61 & 1 & -20.47 & 79.3 & 3 & G0V & & $\cdots$ & $\ldots$ \\
\hline J00482768-7300412 & 04827.69 & $\begin{array}{llll}-73 & 00 & 41.2\end{array}$ & 12.39 & 0.04 & 12.79 & 0.12 & 1 & 107.36 & 65.2 & 1 & B9:V: & A0Ia & 13 & $\mathrm{AzV} 38 ; \mathrm{Sk} 24$ \\
\hline J00483182-7259116 & 04831.83 & $\begin{array}{lll}-72 & 59 & 11.5\end{array}$ & 10.23 & 0.86 & 13.63 & 1.52 & 1 & 121.42 & 113.8 & 2 & & G7Ib & 12 & \\
\hline J00483517-7222102 a & 04835.18 & -722210.3 & 10.09 & 0.88 & 13.58 & 1.65 & 1 & 176.69 & 77.8 & 1 & G2? & G8Iab & 12 & SkKM 32 \\
\hline J00483708-7347222 & 04837.10 & $\begin{array}{lll}-73 & 47 & 22.3\end{array}$ & 11.25 & 0.28 & 12.57 & 0.53 & 1 & 1.46 & 40 & 3 & & & & \\
\hline J00484483-7320190 & 04844.86 & -732018.9 & 8.03 & 0.73 & 10.75 & & 3 & 3.36 & 124 & 3 & K2III & K2III/IV & 22 & Flo 208 \\
\hline J00484542-7255274 & 04845.44 & $\begin{array}{lll}-72 & 55 & 27.4\end{array}$ & 10.63 & 0.86 & 14.11 & 1.51 & 1 & 136.62 & 73.3 & 1 & $\cdots$ & G8Iab & 12 & $\cdots$ \\
\hline J00484775-7304569 a & 04847.75 & $\begin{array}{llll}-73 & 04 & 57.0\end{array}$ & 10.41 & 0.54 & 12.6 & 0.9 & 1 & 32.2 & 110.5 & 3 & G5V & G6III & 12 & $\ldots$ \\
\hline J00484835-7239005 & 04848.37 & -723900.6 & 10.62 & 0.89 & 13.94 & 1.45 & 1 & 136.49 & 66.3 & 1 & $\ldots$ & $\ldots$ & $\ldots$ & SkKM 34 \\
\hline J00485433-7332057 & 04854.36 & -733205.8 & 11.5 & 0.36 & 12.96 & 0.58 & 1 & -9.86 & 66.4 & 3 & $\ldots$ & $\ldots$ & $\ldots$ & $\ldots$ \\
\hline J00490129-7230266 & 04901.32 & -723026.7 & 10.25 & 0.24 & 11.29 & $\ldots$ & 3 & 31.59 & 40.3 & 3 & A8I & $\mathrm{A} 3 \mathrm{~V}$ & 6 & Flo 211 \\
\hline J00490294-7150393 & 04902.96 & -715039.2 & 9.59 & 0.71 & 12.34 & $\ldots$ & 3 & -28.57 & 90 & 3 & K2:III & & $\ldots$ & $\ldots$ \\
\hline J00490296-7321409 & 04902.97 & -732140.9 & 11.05 & 0.03 & 11.02 & -0.04 & 2 & 137.36 & 66.7 & 1 & B2I & B5Ia & 7 & HD $4862 ;$ Sk $27 ;$ RMC 5 \\
\hline J00490802-7217461 a & 04908.02 & $-72 \quad 1746.3$ & 8.97 & 0.55 & 11.18 & & 3 & 29.16 & 121.7 & 3 & KOV & $\mathrm{KOV}$ & 12 & Flo 213 \\
\hline J00490961-7219017 & 04909.62 & -721901.8 & 9.45 & 0.67 & 12 & 1.12 & 1 & -36.31 & 106.7 & 3 & K2III & KOIII & 12 & \\
\hline J00491181-7241346 & 04911.83 & -724134.7 & 10.05 & 0.87 & 13.45 & 1.58 & 1 & 140.99 & 68.7 & 1 & G2I & $\ldots$ & $\cdots$ & SkKM 40 \\
\hline J00491408-7204322 & 04914.10 & -720432.4 & 9.63 & 0.91 & 13.2 & 1.65 & 1 & 155.03 & 89.6 & 1 & G8III & & $\ldots$ & SkKM 42 \\
\hline J00492430-7326449 a & 04924.32 & -732645.0 & 9.89 & 0.42 & 11.63 & $\cdots$ & 3 & 30 & 120.3 & 3 & G5V & $\cdots$ & & $\ldots$ \\
\hline J00493436-7217233 a & 04934.37 & -721723.5 & 11.38 & 0.43 & 13.15 & 0.66 & 1 & 109.36 & 77 & 3 & G0 & G0 & 3 & $2 \mathrm{dFS} 0733$ \\
\hline J00494543-7252477 & 04945.44 & -725247.7 & 12.32 & 0.06 & 12.77 & 0.18 & 1 & 144.12 & 48.9 & 1 & A0:I: & A0I & 8 & $\mathrm{AzV} 53$ \\
\hline J00494643-7227462 & 04946.42 & -722746.3 & 12.26 & 0.05 & 12.66 & 0.11 & 1 & 198.69 & 42.2 & 1 & A3III & & $\ldots$ & $\ldots$ \\
\hline J00495125-7255452 & 04951.27 & -725545.2 & 11.02 & 0.06 & 11.16 & 0 & 2 & 135.92 & 55.2 & 1 & B2I & B2.5Ia & 7 & $\mathrm{AzV} 56 ; \mathrm{Sk} 31$ \\
\hline J00495574-7302508 & 04955.74 & -730250.8 & 10.38 & 0.82 & 13.53 & 1.21 & 1 & 141.56 & 68.2 & 1 & $\ldots$ & & $\ldots$ & SkKM 50 \\
\hline J00495575-7353183 & 04955.75 & $\begin{array}{lll}-73 & 53 & 18.3\end{array}$ & 10.64 & 0.58 & 12.88 & 0.87 & 1 & 2.86 & 34.3 & 3 & & $\mathrm{~K} 2 \mathrm{~V}$ & 12 & $\ldots$ \\
\hline J00495705-7158295 & 04957.04 & $\begin{array}{lll}-71 & 58 & 29.6\end{array}$ & 11.44 & 0.46 & 13.36 & 0.86 & 4 & 25.93 & 93.2 & 3 & G8III & $\cdots$ & & $\cdots$ \\
\hline J00500252-7245241 & 05002.52 & -724524.2 & 10.9 & 0.3 & 12.2 & 0.51 & 1 & 16.89 & 84.4 & 3 & GOI & F9III & 12 & $\ldots$ \\
\hline J00500608-7307452 a & 05006.08 & $\begin{array}{lll}-73 & 07 & 45.3\end{array}$ & 10.46 & 0.14 & 11.02 & 0.13 & 2 & 173.6 & 64.1 & 1 & B5I & $\mathrm{B} 8 \mathrm{Ia}+$ & 7 & HD 4976; Sk 33; RMC 6 \\
\hline J00501135-7211229 & $0 \quad 50 \quad 11.35$ & -721123.0 & 10.14 & 0.87 & 13.49 & 1.55 & 1 & 119.93 & 106.5 & 2 & G5? & $\ldots$ & $\cdots$ & SkKM 53 \\
\hline J00501239-7309580 a & 05012.38 & $\begin{array}{lll}-73 & 09 & 58.1\end{array}$ & 11.09 & 0.39 & 12.73 & 0.67 & 1 & 1.2 & 73.6 & 3 & G5V & & $\cdots$ & $\ldots$ \\
\hline J00501256-7129348 & 05012.56 & -712934.9 & 8.26 & 0.32 & 9.54 & 0.55 & 2 & 22.47 & 124.6 & 3 & $\ldots$ & F7V & 9 & HD 4969 \\
\hline J00501967-7336112 a & 05019.63 & $\begin{array}{lll}-73 & 36 & 11.2\end{array}$ & 6.85 & 0.91 & 10.18 & $\ldots$ & 3 & 55.5 & 110 & 3 & $\ldots$ & $\mathrm{K} 3 \mathrm{~V}$ & 6 & Flo 229; HD 5003 \\
\hline J00502124-7306094 & 05021.25 & -730609.5 & 10.37 & 0.85 & 13.69 & 1.53 & 1 & 135.76 & 76.6 & 1 & $\cdots$ & $\ldots$ & $\ldots$ & ... \\
\hline J00502177-7126485 & 05021.78 & -712648.6 & 11.31 & 0.29 & 12.57 & 0.38 & 5 & 1.87 & 112.9 & 3 & $\cdots$ & $\ldots$ & $\ldots$ & $\cdots$ \\
\hline J00502480-7200011 & 05024.80 & -720001.3 & 9.89 & 0.87 & 13.37 & 1.62 & 4 & 163.13 & 84 & 1 & $\ldots$ & . & $\ldots$ & SkKM 55 \\
\hline J00502879-7245092 a & 05028.79 & -724509.2 & 9.88 & 0.46 & 11.63 & 0.63 & 1 & 119.09 & 91.8 & 2 & $\cdots$ & G0Ib & 15 & RMC 7; cepheid \\
\hline J00503106-7252207 & 05031.07 & $\begin{array}{lll}-72 & 52 & 20.7\end{array}$ & 11.27 & 0.41 & 12.9 & 0.6 & 1 & 150.12 & 41.4 & 1 & $\cdots$ & $\mathrm{O} 5.5 \mathrm{I}(\mathrm{f})$ & 28 & Sk 38 \\
\hline J00503158-7328425 a & 05031.58 & -732842.6 & 10.79 & 0.1 & 11.18 & 0.11 & 2 & 169.3 & 69.8 & 1 & $\mathrm{~A} 2 \mathrm{I}$ & $\mathrm{B} 9 \mathrm{Ia}+$ & 7 & HD 5030; Sk 39; RMC 8 \\
\hline J00503368-7315249 & 05033.68 & -731524.9 & 10.05 & 0.44 & 11.94 & 0.78 & 1 & 167.04 & 57.1 & 1 & $\ldots$ & $\ldots$ & $\ldots$ & $\ldots$ \\
\hline J00503655-7229108 & 05036.55 & -722910.8 & 11.46 & 0.25 & 12.64 & 0.52 & 1 & 19.59 & 96.5 & 3 & $\mathrm{~F} 8 \mathrm{~V}$ & & $\ldots$ & $\ldots$ \\
\hline J00503839-7328182 a & 05038.40 & $\begin{array}{lll}-73 & 28 & 18.2\end{array}$ & 11.03 & 0.04 & 11.04 & -0.02 & 2 & 165.1 & 59.2 & 1 & B2:I: & B1Ia+ & 12 & HD 5045; AzV 78; RMC \\
\hline J00504262-7236481 & 05042.60 & -723648.1 & 10.57 & 0.63 & 12.98 & 0.98 & 1 & 11.99 & 79.8 & 3 & $\ldots$ & G7IV & 12 & \\
\hline J00504717-7242576 a & 05047.16 & -724257.6 & 8.92 & 0.92 & 12.66 & 1.69 & 1 & 136.09 & 67 & 1 & $\cdots$ & $\mathrm{K} 1 \mathrm{Ia} / \mathrm{Iab}$ & 22 & SkKM 58 \\
\hline J00504958-7241541 & 05049.58 & -724154.1 & 10.03 & 0.9 & 13.49 & 1.47 & 1 & 113.99 & 87.3 & 2 & $\cdots$ & G8Ia/Iab & 22 & SkKM 62 \\
\hline J00505473-7337239 & 05054.73 & -733724.0 & 11.28 & 0.25 & 12.53 & 0.48 & 1 & -23.54 & 79.9 & 3 & $\ldots$ & A7V & 6 & Flo 237 \\
\hline J00505814-7138174 & 05058.13 & $\begin{array}{lll}-71 & 38 & 17.4\end{array}$ & 10 & 0.66 & 12.55 & $\ldots$ & 3 & 13.17 & 87.3 & 3 & $\ldots$ & $\ldots$ & $\ldots$ & $\ldots$ \\
\hline J00505824-7241343 & 05058.25 & -724134.2 & 10.29 & 0.35 & 11.87 & $\ldots$ & 3 & -15.22 & 52.3 & 3 & $\ldots$ & GOV & 12 & $\ldots$ \\
\hline
\end{tabular}


Table 3-Continued

\begin{tabular}{|c|c|c|c|c|c|c|c|c|c|c|c|c|c|c|}
\hline 2MASS & $\alpha(\mathrm{J} 2000)$ & $\delta(\mathrm{J} 2000)$ & $K$ & $J-K$ & V & $B-V$ & Ref. $^{\text {b }}$ & $\begin{array}{c}\mathrm{Vel} \\
\left(\mathrm{km} \mathrm{s}^{-1}\right)\end{array}$ & $r^{\mathrm{c}}$ & Category $^{\mathrm{d}}$ & $\begin{array}{c}\text { Hydra } \\
\text { Class }\end{array}$ & $\begin{array}{c}\text { Literature } \\
\text { Class }\end{array}$ & Ref. $^{e}$ & Comments $^{f}$ \\
\hline J00510268-7236448 & 05102.69 & -723644.9 & 11.2 & 0.37 & 12.7 & 0.6 & 1 & 7.89 & 94.6 & 3 & G0V & $\ldots$ & $\ldots$ & . \\
\hline J00511191-7228177 & 05111.91 & $-72 \quad 28 \quad 17.8$ & 12.06 & 0.12 & 12.54 & 0.08 & 1 & 141.09 & 39.5 & 1 & $\mathrm{~A} 2 \mathrm{I}$ & $\mathrm{A} 2 \mathrm{I}$ & 8 & $\mathrm{AzV} 90 ;$ Sk 44 \\
\hline J00511365-7233016 & $051 \quad 13.65$ & $\begin{array}{lll}-72 & 3301.6\end{array}$ & 10.73 & 0.81 & 13.87 & 1.41 & 1 & 144.89 & 92.1 & 1 & $\ldots$ & G8Ib & 12 & SkKM 68 \\
\hline J00511592-7135136 & 05115.92 & -713513.6 & 10.26 & 0.9 & 13.8 & 1.46 & 5 & 153.97 & 92.8 & 1 & $\ldots$ & $\ldots$ & $\cdots$ & $\ldots$ \\
\hline J00511737-7133589 & 05117.38 & -713358.8 & 9.93 & 0.41 & 11.52 & & 3 & 36.27 & 123.2 & 3 & $\ldots$ & & $\ldots$ & $\ldots$ \\
\hline J00511824-7243246 & 05118.25 & $\begin{array}{lll}-72 & 43 & 24.7\end{array}$ & 10.59 & 0.86 & 13.77 & 1.33 & 1 & 150.79 & 108.7 & 1 & $\ldots$ & & $\ldots$ & \\
\hline J00513037-7316098 & 05130.37 & $\begin{array}{lll}-73 & 16 & 09.9\end{array}$ & 11.76 & 0.07 & 12.13 & 0.07 & 2 & 159.26 & 56.9 & 1 & AOI & B9Ia & 7 & $\mathrm{AzV} 101 ; \mathrm{Sk} 47$ \\
\hline J00513209-7255507 & 05132.09 & -725550.7 & 11.01 & 0.45 & 12.73 & 0.59 & 1 & 142.46 & 58.8 & 1 & A $5: I ?$ & & & \\
\hline J00513554-7219580 & 05135.52 & $\begin{array}{lll}-72 & 19 & 58.1\end{array}$ & 8.97 & 0.68 & 11.51 & 1.09 & 1 & 32.99 & 115 & 3 & G8III & KoIII & 12 & \\
\hline J00513658-7355459 & 05136.56 & -735545.9 & 8.57 & 0.72 & 11.15 & 1.14 & 1 & 59.76 & 86.4 & 3 & & KOIII & 12 & Flo 246 \\
\hline J00513764-7225596 & 05137.64 & -722559.6 & 9.57 & 0.88 & 12.97 & 1.64 & 1 & 155.59 & 84.1 & 1 & $\ldots$ & G8.5Iab & 12 & SkKM 76 \\
\hline J00513784-7240061 & 05137.85 & $\begin{array}{lll}-72 & 40 & 06.2\end{array}$ & 10.67 & 0.56 & 12.83 & 0.74 & 1 & 145.69 & 49.4 & 1 & F8V V & & $\ldots$ & $\ldots$ \\
\hline J00513860-7131570 & 05138.61 & $\begin{array}{llll}-71 & 31 & 57.1\end{array}$ & 7.38 & 0.69 & 10.02 & $\ldots$ & 3 & -18.33 & 93.3 & 3 & & Ko & 9 & HD 5115 \\
\hline J00515197-7244135 & 05151.98 & -724413.5 & 11.72 & 0.04 & 12.13 & 0.08 & 2 & 147.09 & 57.9 & 1 & A3Ia & A0Ia & 13 & $\mathrm{AzV} 110 ; \mathrm{Sk} 49$ \\
\hline J00515354-7223510 & 05153.54 & -722351.0 & 10.45 & 0.63 & 12.88 & 1 & 1 & -23.61 & 99 & 3 & G5V & & & \\
\hline J00515545-7248584 & 05155.47 & -724858.3 & 11.09 & 0.45 & 12.66 & 0.55 & 1 & -27.41 & 71.3 & 3 & GOIV & F0 & 11 & $\mathrm{AzV} 30 \mathrm{~F}$ \\
\hline J00515983-7245596 & 05159.84 & -724559.7 & 12.22 & 0.15 & 12.8 & 0.11 & 1 & 118.29 & 40.7 & 1 & A3I & $\mathrm{A} 1$ & 11 & $\mathrm{AzV} 32 \mathrm{~F}$ \\
\hline J00515998-7303332 & 05159.98 & $\begin{array}{llll}-73 & 03 & 33.3\end{array}$ & 10.65 & 0.86 & 13.72 & 1.15 & 1 & 150.86 & 110.3 & 1 & $\ldots$ & $\cdots$ & $\cdots$ & $\cdots$ \\
\hline J00520744-7333007 & 05207.46 & -733300.8 & 11.05 & 0.4 & 12.63 & 0.75 & 1 & 55.16 & 101.4 & 3 & $\ldots$ & & $\ldots$ & $\ldots$ \\
\hline J00520803-7216491 a & 05208.03 & $\begin{array}{lll}-72 & 16 & 49.3\end{array}$ & 10.61 & 0.89 & 13.96 & 1.43 & 1 & 154.83 & 75.8 & 1 & $\cdots$ & $\mathrm{K} 1 \mathrm{Ib}$ & 12 & SkKM 83 \\
\hline J00521105-7320365 & 05211.05 & -732036.6 & 11.85 & 0.17 & 12.53 & 0.19 & 1 & 143.36 & 44.6 & 1 & A2:I & $\ldots$ & $\ldots$ & $\ldots$ \\
\hline J00521331-7334478 & 05213.32 & -733448.0 & 11.59 & 0.32 & 12.92 & 0.57 & 1 & 39.96 & 73.3 & 3 & & & $\cdots$ & $\cdots$ \\
\hline J00521343-7253209 & 05213.43 & -725321.0 & 8.55 & 0.67 & 11.03 & 1.07 & 1 & 16.76 & 95.5 & 3 & KoIII & K1III/IV & 22 & Flo 255 \\
\hline J00521905-7309229 & 05219.06 & $\begin{array}{lll}-73 & 09 & 23.2\end{array}$ & 10.74 & 0.77 & 13.26 & 0.66 & 1 & 138.26 & 72.9 & 1 & $\ldots$ & G0Iab & 12 & $\ldots$ \\
\hline J00522498-7241029 & 05224.98 & -724103.2 & 12.4 & 0.1 & 12.69 & 0.03 & 1 & 150.09 & 45.2 & 1 & B5I & B8I & 8 & $\mathrm{AzV} 122$ \\
\hline J00522757-7318563 & 05227.57 & -731856.4 & 12.46 & 0.1 & 12.54 & 0.03 & 1 & 160.56 & 48.1 & 1 & $\mathrm{~B} 2 \mathrm{I}$ & B3Ia & 7 & $\mathrm{AzV} 125 ; \mathrm{Sk} 52$ \\
\hline J00523079-7302581 & 05230.80 & $-7302 \quad 58.2$ & 9.39 & 0.64 & 11.81 & 1.02 & 1 & 50.46 & 106 & 3 & KOIII & KOIII & 12 & \\
\hline J00523081-7313144 & 05230.82 & -731314.6 & 11.44 & 0.25 & 12.42 & 0.27 & 1 & 127.86 & 41.3 & 1 & $\mathrm{~A} 2: \mathrm{I}$ & F2I & 8 & $\mathrm{AzV} 127$ \\
\hline J00523150-7211374 a & 05231.49 & -721137.5 & 9.41 & 0.89 & 12.98 & 1.65 & 1 & 160.13 & 85.3 & 1 & $\ldots$ & G8Iab & 12 & SkKM 91 \\
\hline J00523564-7251053 & 05235.65 & -725105.3 & 9.74 & 0.85 & 13.04 & 1.5 & 1 & 129.19 & 80.5 & 1 & K0:III: & ... & $\ldots$ & SkKM 94 \\
\hline J00524734-7320299 & 05247.37 & -732030.0 & 11.38 & 0.37 & 12.95 & 0.67 & 1 & 0.66 & 73.9 & 3 & $\mathrm{G} 2 \mathrm{~V}$ & $\ldots$ & & $\ldots$ \\
\hline J00525020-7213199 a & 05250.19 & -721320.0 & 10.41 & 0.89 & 13.76 & 1.5 & 1 & 151.23 & 94.2 & 1 & $\ldots$ & $\ldots$ & $\ldots$ & $\ldots$ \\
\hline J00525121-7306535 & 05251.23 & -730653.6 & 10.42 & 0.12 & 10.95 & 0.13 & 2 & 137.86 & 61.3 & 1 & A2I & A0Ia & 10 & HD 5277; Sk 54; RMC 1 \\
\hline J00525704-7212000 a & 05257.05 & -721200.0 & 10.79 & 0.54 & 12.88 & 0.82 & 1 & 32.93 & 109.1 & 3 & KOV & & & \\
\hline J00530146-7223591 & 05301.46 & $\begin{array}{lll}-72 & 23 & 59.2\end{array}$ & 10.51 & 0.83 & 13.61 & 1.27 & 1 & 150.39 & 93 & 1 & $\ldots$ & G2 & 3 & $2 \mathrm{dFS} 0905$ \\
\hline J00530165-7230305 & 05301.65 & -723030.6 & 10.67 & 0.79 & 13.68 & 1.35 & 1 & 138.29 & 77.3 & 1 & $\cdots$ & $\cdots$ & $\cdots$ & SkKM 99 \\
\hline J00530229-7154161 & 05302.29 & -715416.1 & 11.29 & 0.38 & 12.92 & & 3 & 10.36 & 86.4 & 3 & $\ldots$ & & $\ldots$ & \\
\hline J00530489-7238000 & 05304.89 & -723800.1 & 10.57 & 0.06 & 10.85 & 0.04 & 2 & 152.44 & 48.2 & 1 & $\ldots$ & B8Ia+ & 7 & HD 5291; Sk 56; RMC 1 \\
\hline J00530773-7307095 & 05307.74 & -730709.6 & 12.45 & 0.02 & 12.65 & 0.06 & 1 & 153.76 & 52.2 & 1 & B8:I: & $\ldots$ & $\ldots$ & \\
\hline J00530894-7229386 & 05308.94 & -722938.7 & 8.45 & 0.91 & 11.91 & 1.77 & 1 & 133.29 & 75.2 & 1 & G5I & K3Ia & 12 & SkKM 104 \\
\hline J00530985-7313248 & 05309.86 & $\begin{array}{lll}-73 & 1324.9\end{array}$ & 9.61 & 0.31 & 11.01 & & 3 & 8.76 & 105.3 & 3 & GOV & F9III & 12 & Flo 270 \\
\hline J00531104-7257039 & 05311.07 & -725703.9 & 9.58 & 0.69 & 12.19 & 1.18 & 1 & 1.46 & 104.2 & 3 & K1III & K1III/IV & 22 & \\
\hline J00531572-7222029 & 05315.72 & $\begin{array}{lll}-72 & 22 & 03.0\end{array}$ & 12 & 0.19 & 12.79 & 0.21 & 1 & 130.29 & 38.2 & 1 & A5I & F5I? & 8 & $\mathrm{AzV} 142$ \\
\hline J00531596-7315325 & 05315.96 & $-73 \quad 1532.6$ & 12.19 & 0.14 & 12.82 & 0.19 & 1 & 158.86 & 50.6 & 1 & A3:II: & A5 & 11 & $\mathrm{AzV} 37 \mathrm{~F}$ \\
\hline J00532140-7217166 a & 05321.41 & $\begin{array}{llll}-72 & 17 & 16.7\end{array}$ & 10.98 & 0.35 & 12.35 & 0.5 & 1 & 168.43 & 36.2 & 1 & FOI & & $\ldots$ & \\
\hline J00532362-7247013 a & 05323.61 & $\begin{array}{llll}-72 & 4701.4\end{array}$ & 10.12 & 0.82 & 13.32 & 1.21 & 1 & 155.52 & 88.3 & 1 & & G8Iab & 12 & SkKM 108 \\
\hline J00532650-7251595 a & 05326.51 & -725159.6 & 10.24 & 0.71 & 12.85 & 1.11 & 1 & 24.22 & 87.9 & 3 & G8I & K1III & 12 & $\ldots$ \\
\hline J00533093-7231577 & 05330.92 & -723157.9 & 10.2 & 0.89 & 13.61 & 1.56 & 1 & 136.99 & 73.7 & 1 & $\ldots$ & $\ldots$ & $\ldots$ & $\ldots$ \\
\hline J00533726-7223557 & 05337.26 & -722355.8 & 12.22 & 0.19 & 12.99 & 0.23 & 1 & 106.49 & 35.6 & 3 & A5I & $\cdots$ & $\cdots$ & $\cdots$ \\
\hline J00533784-7232393 & 05337.82 & $\begin{array}{l}-72 \quad 3239.4 \\
\end{array}$ & 11.56 & 0.29 & 12.7 & 0.34 & 1 & 167.89 & 29.7 & 1 & FOI & $\ldots$ & $\ldots$ & $\ldots$ \\
\hline
\end{tabular}


Table 3-Continued

\begin{tabular}{|c|c|c|c|c|c|c|c|c|c|c|c|c|c|c|}
\hline 2MASS & $\alpha(\mathrm{J} 2000)$ & $\delta(\mathrm{J} 2000)$ & K & $J-K$ & V & $B-V$ & Ref. $^{\text {b }}$ & $\begin{array}{c}\mathrm{Vel} \\
\left(\mathrm{km} \mathrm{s}^{-1}\right)\end{array}$ & $r^{\mathrm{c}}$ & Category $^{\mathrm{d}}$ & $\begin{array}{c}\text { Hydra } \\
\text { Class }\end{array}$ & $\begin{array}{c}\text { Literature } \\
\text { Class }\end{array}$ & Ref. $^{\mathrm{e}}$ & Comments $^{f}$ \\
\hline J00534149-7352097 & 05341.51 & $\begin{array}{lll}-73 & 52 & 09.9\end{array}$ & 7.19 & 0.3 & 8.44 & 0.51 & 2 & 12.36 & 81.6 & 3 & $\ldots$ & F6IV & 9 & Flo 279; HD 5370 \\
\hline J00534262-7217135 & 05342.65 & $\begin{array}{llll}-72 & 17 & 13.7\end{array}$ & 10.28 & 0.41 & 11.89 & 0.59 & 1 & 143.99 & 77.6 & 1 & G2:I & $\mathrm{F} 2 \mathrm{Ib}$ & 15 & RMC 12 \\
\hline J00534575-7253389 & 05345.76 & -725339.0 & 9.62 & 0.81 & 12.94 & 1.57 & 1 & 141.64 & 67.7 & 1 & $\ldots$ & G6Iab & 12 & SkKM 111 \\
\hline J00535656-7254396 & 05356.56 & -725439.6 & 10.49 & 0.9 & 13.63 & 1.12 & 1 & 135.64 & 56.2 & 1 & & G8II & 12 & SkKM 118 \\
\hline J00535761-7228404 & 05357.59 & -722840.5 & 12.58 & 0.02 & 12.62 & 0.01 & 1 & 142.79 & 46.5 & 1 & B8I & B9I & 8 & $\mathrm{AzV} 150$ \\
\hline J00540272-7315318 & 05402.71 & $\begin{array}{lll}-73 & 15 & 32.0\end{array}$ & 12.17 & 0.21 & 12.92 & 0.2 & 1 & 116.66 & 35.8 & 1 & $\ldots$ & & & . \\
\hline J00540321-7231445 & 05403.21 & -723144.7 & 11.17 & 0.14 & 11.87 & 0.17 & 2 & 117.54 & 41.8 & 1 & $\cdots$ & A3I & 14 & $\mathrm{AzV}$ 152; Sk 58 \\
\hline J00540520-7426579 & 05405.20 & -742657.9 & 8 & 0.62 & 10.35 & & 3 & -6.41 & 56.5 & 3 & $\cdots$ & K1 & 6 & Flo 290 \\
\hline J00541781-7322539 & 05417.82 & -732254.1 & 9.67 & 0.67 & 12.2 & 1.02 & 1 & 27.56 & 151.9 & 3 & G8 & K1III/IV & 22 & \\
\hline J00542237-7328093 & 05422.39 & $\begin{array}{lll}-73 & 28 & 09.3\end{array}$ & 10.64 & 0.29 & 11.84 & 0.49 & 1 & 5.83 & 28.2 & 3 & F5? & & & \\
\hline J00542687-7252596 & 05426.88 & $-7252 \quad 59.8$ & 9.49 & 0.84 & 12.84 & 1.68 & 1 & 150.84 & 53.6 & 1 & $\cdots$ & G7.5Iab & 12 & SkKM 123 \\
\hline J00543871-7303076 & 05438.71 & $\begin{array}{llll}-73 & 03 & 07.8\end{array}$ & 10.83 & 0.23 & 12.9 & $\ldots$ & 3 & -18.04 & 69.7 & 3 & FoIII & F9V & 12 & $\mathrm{AzV} 47 \mathrm{~F}$ \\
\hline J00543893-7221199 & 05438.96 & $\begin{array}{llll}-72 & 21 & 19.8\end{array}$ & 9.8 & 0.91 & 13.32 & 1.58 & 1 & 145.39 & 83.3 & 1 & $\cdots$ & G8Ia/Iab & 22 & SkKM 127 \\
\hline J00544146-7214139 a & 05441.47 & $\begin{array}{lll}-72 & 14 & 13.9\end{array}$ & 12.73 & 0.07 & 12.96 & 0.05 & 1 & 149.43 & 48.6 & 1 & B8I & $\ldots$ & $\cdots$ & $\ldots$ \\
\hline J00544483-7325111 & 05444.86 & -732511.2 & 8.53 & 0.71 & 11.18 & 1.27 & 1 & 35.46 & 80.4 & 3 & KoIII: & K0.5III & 12 & $\ldots$ \\
\hline J00545086-7341274 & 05450.86 & -734127.6 & 8.78 & 0.76 & 11.5 & 1.18 & 1 & 39.53 & 90.6 & 3 & KOIII & K1III/IV & 22 & $\ldots$ \\
\hline J00545150-7322073 & 05451.53 & -732207.5 & 11.07 & 0.34 & 12.55 & 0.64 & 1 & 19.66 & 105.1 & 3 & G0:V & & $\cdots$ & $\cdots$ \\
\hline J00545294-7247208 a & 05452.95 & -724721.0 & 11.08 & 0.4 & 12.76 & 0.68 & 1 & 42.02 & 83.5 & 3 & $\mathrm{G} 2 \mathrm{~V}$ & & & \\
\hline J00545445-7309033 & 05454.45 & $\begin{array}{llll}-73 & 09 & 03.6\end{array}$ & 10.29 & 0.7 & 13.12 & 1.38 & 1 & 154.46 & 99.4 & 1 & & G5Ia/Iab & 22 & SkKM 129 \\
\hline J00545575-7342543 a & 05455.77 & $-7342 \quad 54.4$ & 8.47 & 0.35 & 9.96 & $\ldots$ & 3 & 29.39 & 81.7 & 3 & G8V & G1V & 12 & Flo 304; HD 5498 \\
\hline J00545763-7304401 & 05457.63 & -730440.3 & 11.38 & 0.33 & 12.68 & 0.46 & 1 & 172.56 & 34.9 & 1 & A5I & $\ldots$ & $\ldots$ & $\ldots$ \\
\hline J00550182-7206498 & 05501.82 & -720649.8 & 8.2 & 0.59 & 10.58 & $\ldots$ & 3 & 7.16 & 110.4 & 3 & $\ldots$ & G & 6 & Flo 302 \\
\hline J00550377-7300367 & $0 \quad 55 \quad 03.77$ & -730036.9 & 8.86 & 0.87 & 12.4 & 1.75 & 1 & 163.66 & 76.1 & 1 & $\ldots$ & G6Ia/Iab & 22 & SkKM 132 \\
\hline J00550944-7230556 & $055 \quad 09.44$ & -723055.7 & 10.52 & 0.85 & 13.7 & 1.42 & 1 & 148.74 & 90.9 & 1 & & G7.5Iab & 12 & $\ldots$ \\
\hline J00551077-7227095 & $055 \quad 10.76$ & -722709.6 & 11.39 & 0.27 & 12.56 & 0.43 & 1 & 166.99 & 29.9 & 1 & FOI & $\ldots$ & $\ldots$ & $\ldots$ \\
\hline J00551256-7226111 a & 05512.56 & -722611.1 & 11.6 & 0.24 & 12.58 & 0.3 & 1 & 169.42 & 26.1 & 1 & FOI & $\cdots$ & $\cdots$ & \\
\hline J00551277-7230424 & $055 \quad 12.77$ & -723042.4 & 10.46 & 0.88 & 13.67 & 1.35 & 1 & 153.19 & 98.8 & 1 & & & $\ldots$ & SkKM 134 \\
\hline J00551819-7314205 & $0 \quad 55 \quad 18.21$ & -731420.8 & 12.25 & 0.02 & 12.41 & 0.03 & 1 & 153.26 & 55.1 & 1 & A0I & A0I & 13 & $\mathrm{AzV} 166 ; \mathrm{Sk} 60$ \\
\hline J00552236-7216385 & 05522.37 & -721638.5 & 10.19 & 0.89 & 13.61 & 1.51 & 1 & 152.93 & 73.3 & 1 & $\cdots$ & G7.5Iab & 12 & SkKM 135/6 \\
\hline J00552265-7205218 & 05522.65 & -720521.8 & 11.31 & 0.29 & 12.54 & 0.4 & 1 & 182.26 & 34.9 & 1 & $\ldots$ & $\ldots$ & $\cdots$ & ... \\
\hline J00552776-7243454 a & 05527.77 & -724345.6 & 11.74 & 0.17 & 12.54 & 0.21 & 1 & 117.32 & 35.2 & 1 & A5I & - & $\ldots$ & \\
\hline J00553015-7228496 a & 05530.14 & -722849.7 & 10.53 & 0.89 & 13.78 & 1.34 & 1 & 154.42 & 83 & 1 & $\cdots$ & G8Ia/Iab & 22 & $\ldots$ \\
\hline J00553056-7334291 & 05530.57 & -733429.3 & 9.17 & 0.32 & 10.51 & $\cdots$ & 3 & -16.54 & 81.6 & 3 & & F9IV & 12 & HD 5549 \\
\hline J00553664-7239157 & 05536.64 & -723915.9 & 10.7 & 0.29 & 12.07 & 0.57 & 1 & 31.69 & 129.5 & 3 & GoIII & & $\ldots$ & $\ldots$ \\
\hline J00554163-7209429 & 05541.65 & -720942.8 & 11.42 & 0.3 & 12.73 & 0.53 & 1 & 17.96 & 66.7 & 3 & $\ldots$ & $\ldots$ & & $\ldots$ \\
\hline J00554756-7416386 & 05547.56 & -741638.7 & 11.53 & 0.28 & 12.87 & & 3 & -23.15 & 68.1 & 3 & $\cdots$ & & $\ldots$ & $\ldots$ \\
\hline J00555119-7346230 & 05551.20 & -734623.2 & 9.79 & 0.81 & 12.8 & 1.26 & 1 & 61.39 & 118.9 & 3 & KoIII & K2III & 12 & $\ldots$ \\
\hline J00555508-7240305 & 05555.07 & -724030.6 & 9.34 & 0.65 & 12.02 & 1.32 & 1 & 160.49 & 77.3 & 1 & $\ldots$ & G3.5Ia & 12 & \\
\hline J00560334-7229081 & 05603.35 & -722908.0 & 10.96 & 0.33 & 12.32 & 0.56 & 1 & -2.28 & 106.9 & 3 & $\cdots$ & F8V & 17 & $\mathrm{Cl}^{*} \mathrm{NGC} 330 \mathrm{Arp} \mathrm{i}$ \\
\hline J00561519-7152311 & 05615.19 & $\begin{array}{lll}-71 & 52 & 31.2\end{array}$ & 10.31 & 0.92 & 13.81 & 1.62 & 1 & 157.36 & 93.2 & 1 & $\cdots$ & KOIab & 12 & SkKM 147 \\
\hline J00562256-7228359 & 05622.58 & -722835.8 & 11.9 & 0.17 & 12.31 & 0.11 & 1 & 132.64 & 43.9 & 1 & $\ldots$ & A5I & 17 & $\mathrm{Cl}^{*} \mathrm{NGC} 330$ Arp 9 \\
\hline J00562354-7252534 & 05623.55 & -725253.5 & 10.5 & 0.37 & 12.02 & 0.66 & 1 & 49.84 & 109.7 & 3 & $\cdots$ & G1V & 12 & $\cdots$ \\
\hline J00562643-7328232 & 05626.43 & -732823.3 & 9.62 & 0.88 & 12.74 & 1.66 & 1 & 164.03 & 59.7 & 1 & & K4Ia/Iab & 22 & SkKM 152 \\
\hline J00562700-7409243 & 05627.00 & -740924.3 & 9.09 & 0.68 & 11.65 & $\ldots$ & 3 & 28.65 & 116 & 3 & $\ldots$ & & $\cdots$ & $\ldots$ \\
\hline J00562776-7330270 & 05627.75 & -733027.2 & 10.43 & 0.86 & 13.63 & 1.5 & 1 & 164.23 & 39.9 & 1 & & G8Iab & 12 & $\ldots$ \\
\hline J00563534-7336391 & 05635.34 & -733639.2 & 8.91 & 0.73 & 11.49 & 1.11 & 1 & 9.89 & 82.1 & 3 & K2III & & & . \\
\hline J00563633-7226464 a & 05636.34 & -722646.5 & 9.86 & 0.92 & 13.54 & 1.78 & 1 & 144.32 & 84.1 & 1 & $\cdots$ & G8Ia/Iab & 22 & SkKM 155 \\
\hline J00563923-7300523 & 05639.22 & $-7300 \quad 52.4$ & 10.85 & 0.67 & 13.33 & 1.06 & 1 & 74.44 & 33.3 & 2 & $\ldots$ & K1.5III & $\cdots$ & $\ldots$ \\
\hline J00564288-7158579 & 05642.89 & $-7158 \quad 57.6$ & 10.42 & 0.91 & 13.79 & 1.54 & 1 & 160.06 & 78.8 & 1 & $\cdots$ & G8Ib & 12 & SkKM 156 \\
\hline J00565219-7348448 & 05652.18 & -734844.9 & 9.44 & 0.79 & 12.3 & 1.18 & 1 & 11.59 & 84.1 & 3 & K2III & $\ldots$ & $\ldots$ & $\ldots$ \\
\hline
\end{tabular}


Table 3-Continued

\begin{tabular}{|c|c|c|c|c|c|c|c|c|c|c|c|c|c|c|}
\hline 2MASS & $\alpha(\mathrm{J} 2000)$ & $\delta(\mathrm{J} 2000)$ & K & $J-K$ & V & $B-V$ & Ref. $^{b}$ & $\begin{array}{c}\mathrm{Vel} \\
\left(\mathrm{km} \mathrm{s}^{-1}\right)\end{array}$ & $r^{\mathrm{c}}$ & Category $^{\mathrm{d}}$ & $\begin{array}{l}\text { Hydra } \\
\text { Class }\end{array}$ & $\begin{array}{c}\text { Literature } \\
\text { Class }\end{array}$ & Ref. $^{\text {e }}$ & Comments ${ }^{\mathrm{f}}$ \\
\hline J00565449-7258094 & 05654.49 & -725809.6 & 9.01 & 0.64 & 11.37 & 0.99 & 1 & -31.06 & 128.2 & 3 & & $\ldots$ & $\ldots$ & \\
\hline J00570028-7424356 & 05700.26 & -742435.8 & 11.83 & 0.28 & 13.23 & 0.5 & 4 & 22.82 & 82.1 & 3 & & $\ldots$ & $\ldots$ & \\
\hline J00570053-7415257 & 05700.53 & $\begin{array}{llll}-74 & 15 & 25.7\end{array}$ & 10.16 & 0.71 & 12.92 & 1.21 & 5 & 111.32 & 93.4 & 2 & $\ldots$ & & & $\cdots$ \\
\hline J00570451-7229402 & 05704.52 & -722940.2 & 10.74 & 0.82 & 13.84 & 1.28 & 1 & 151.64 & 101.3 & 1 & $\cdots$ & G6.5Ib & 12 & $\cdots$ \\
\hline J00570920-7201181 & 05709.19 & $-7201 \quad 17.8$ & 11.61 & 0.3 & 12.83 & 0.51 & 1 & 4.96 & 66.1 & 3 & & $\ldots$ & $\ldots$ & $\ldots$ \\
\hline J00571085-7332097 & 05710.86 & $-7332 \quad 10.1$ & 7.95 & 0.84 & 10.82 & & 3 & 54.93 & 124 & 3 & G8Ve & & $\ldots$ & . \\
\hline J00571147-7228399 & $057 \quad 11.48$ & -722839.9 & 12.89 & 0.03 & 13.14 & 0.06 & 1 & 158.74 & 35.1 & 1 & $\ldots$ & B9(Iab) & 3 & $\mathrm{AzV} 66 \mathrm{~F}$ \\
\hline J00571148-7129397 & 05711.50 & $\begin{array}{lll}-71 & 29 & 39.7\end{array}$ & 9.26 & 0.57 & 11.48 & $\ldots$ & 3 & 29.5 & 158.7 & 3 & $\ldots$ & $\ldots$ & $\cdots$ & $\ldots$ \\
\hline J00571353-7323288 & $057 \quad 13.54$ & -732328.9 & 11.04 & 0.37 & 12.5 & 0.55 & 1 & 3.63 & 50.8 & 3 & G0V & $\ldots$ & $\ldots$ & \\
\hline J00571770-7242068 & $057 \quad 17.68$ & $-7242 \quad 07.0$ & 11.5 & 0.36 & 13.03 & 0.64 & 1 & -80.56 & 67.7 & 3 & $\ldots$ & $\ldots$ & & $\ldots$ \\
\hline J00572370-7239586 & 05723.67 & -723958.7 & 11.79 & 0.23 & 12.81 & 0.42 & 1 & 25.34 & 54.3 & 3 & $\ldots$ & $\ldots$ & $\ldots$ & \\
\hline J00572384-7256156 & 05723.83 & $\begin{array}{lll}-72 & 56 & 15.8\end{array}$ & 10.21 & 0.59 & 12.42 & 0.92 & 1 & 63.34 & 122.3 & 3 & $\ldots$ & $\ldots$ & $\ldots$ & $\ldots$ \\
\hline J00572982-7421032 & 05729.82 & -742103.4 & 11.51 & 0.26 & 12.73 & 0.02 & 3 & 4.12 & 58.7 & 3 & $\ldots$ & $\ldots$ & & $\ldots$ \\
\hline J00573683-7151251 & 05736.83 & -715125.2 & 9.75 & 0.69 & 12.29 & 1 & 1 & 9.26 & 88.9 & 3 & $\ldots$ & K1IV & 12 & $\ldots$ \\
\hline J00574139-7237523 & 05741.38 & $-7237 \quad 52.2$ & 10.57 & 0.91 & 13.89 & 1.44 & 1 & 142.54 & 76.3 & 1 & $\cdots$ & G7Ib & 12 & $\cdots$ \\
\hline J00574140-7404505 & 05741.40 & -740450.5 & 11.02 & 0.62 & 13.39 & 1.05 & 2 & 3.22 & 74.3 & 3 & $\ldots$ & & to & $\ldots$ \\
\hline J00574622-7242207 & 05746.20 & $-7242 \quad 20.8$ & 12.31 & 0.18 & 12.89 & 0.13 & 1 & 143.24 & 18.6 & 1 & $\ldots$ & $\ldots$ & & $\ldots$ \\
\hline J00575242-7200254 & 05752.42 & -720025.3 & 10.22 & 0.71 & 12.85 & 1.13 & 1 & 3.36 & 97.3 & 3 & & $\ldots$ & $\ldots$ & \\
\hline J00575495-7229451 & 05754.95 & -722945.2 & 10.43 & 0.36 & 11.93 & 0.65 & 1 & 55.49 & 86.3 & 3 & GoIII & $\ldots$ & $\ldots$ & \\
\hline J00575574-7345341 & 05755.73 & -734534.1 & 8.39 & 0.62 & 10.77 & $\ldots$ & 3 & -11.41 & 120.6 & 3 & KOIII & G7III/IV & 22 & Flo 358; HD 5829 \\
\hline J00575689-7333436 & 05756.87 & -733343.8 & 9.84 & 0.7 & 12.76 & 1.42 & 1 & 180.13 & 62.6 & 1 & & G1Ia/Iab & 22 & \\
\hline J00580226-7217566 & $058 \quad 02.28$ & $-72 \quad 17 \quad 56.6$ & 10.58 & 0.29 & 11.86 & 0.45 & 2 & 204.99 & 31.8 & 1 & FOI & F2Ia & 13 & AzV 197 \\
\hline J00580232-7206149 & $0 \quad 58 \quad 02.32$ & $-7206 \quad 14.7$ & 10.6 & 0.92 & 14.02 & 1.66 & 1 & 143.59 & 81.8 & 1 & $\cdots$ & KOIb & 12 & $\ldots$ \\
\hline J00580363-7225261 & $058 \quad 03.63$ & -722526.0 & 10.36 & 0.66 & 12.79 & 1.08 & 1 & 8.91 & 82.8 & 3 & $\ldots$ & & & $\ldots$ \\
\hline J00580791-7238305 & $058 \quad 07.90$ & -723830.5 & 11.85 & 0.06 & 12.14 & 0.07 & 2 & 177.34 & 45.9 & 1 & $\ldots$ & B8Ia & 7 & $\mathrm{AzV} 200 ;$ Sk 69 \\
\hline J00581449-7301127 & $\begin{array}{lll}0 & 58 & 14.49\end{array}$ & $\begin{array}{lll}-73 & 01 & 12.8\end{array}$ & 10.45 & 0.3 & 11.72 & 0.51 & 1 & 16.04 & 84.9 & 3 & $\ldots$ & F5 & 6 & Flo 364 \\
\hline J00581584-7229109 a & $058 \quad 15.84$ & -722910.9 & 11.46 & 0.23 & 12.55 & 0.47 & 1 & 7.21 & 64.9 & 3 & $\mathrm{~F} 2 \mathrm{~V}$ & $\ldots$ & $\ldots$ & $\ldots$ \\
\hline J00581662-7204150 & 05816.63 & -720414.9 & 10.04 & 0.46 & 11.83 & 0.73 & 1 & -9.11 & 85.9 & 3 & G5I & $\mathrm{G} 4.5 \mathrm{~V}$ & 12 & \\
\hline J00582319-7221352 & 05823.21 & -722135.1 & 11.79 & 0.12 & 12.27 & 0.12 & 1 & 148.49 & 37.5 & 1 & A3I & A0I & 13 & $\mathrm{AzV} 205 ; \mathrm{Sk} 71$ \\
\hline J00582953-7211345 & $058 \quad 29.54$ & -721134.6 & 11.47 & 0.31 & 12.63 & 0.43 & 1 & 2.29 & 116.8 & 3 & $\mathrm{~F} 8 \mathrm{~V}$ & & $\cdots$ & ... \\
\hline J00584121-7226154 a & 05841.23 & -722615.5 & 11.08 & 0.12 & 11.51 & 0.1 & 2 & 172.41 & 75.6 & 1 & B8I & A0Ia & 10 & AzV 211; Sk 74 \\
\hline J00584187-7335251 & 05841.88 & -733525.3 & 10.97 & 0.61 & 13.36 & 0.99 & 1 & 27.33 & 54.4 & 3 & & $\mathrm{~K} 2 \mathrm{III} / \mathrm{IV}$ & 22 & \\
\hline J00584242-7209434 & 05842.44 & -720943.4 & 10.71 & 0.39 & 12.16 & 0.59 & 1 & -7.71 & 95 & 3 & G0V & & & \\
\hline J00584479-7300574 & 05844.78 & -730057.6 & 9.97 & 0.3 & 11.26 & $\ldots$ & 3 & -3.46 & 79.3 & 3 & $\ldots$ & F5 & 6 & HD 5913 \\
\hline J00585073-7201179 & 05850.74 & $-7201 \quad 17.8$ & 10.59 & 0.25 & 11.8 & $\ldots$ & 3 & 0.19 & 96.5 & 3 & F8I & $\ldots$ & $\ldots$ & $\ldots$ \\
\hline J00585391-7212048 & 05853.93 & -721204.8 & 9.93 & 0.89 & 13.34 & 1.62 & 1 & 148.19 & 91.2 & 1 & KO:III: & $\ldots$ & $\ldots$ & \\
\hline J00585470-7250596 & 05854.68 & -725059.8 & 10.63 & 0.86 & 13.81 & 1.48 & 1 & 190.44 & 78 & 1 & $\ldots$ & G7Iab & 12 & SkKM 183 \\
\hline J00590884-7401188 & 05908.87 & $-7401 \quad 18.9$ & 11.31 & 0.35 & 12.84 & $\ldots$ & 3 & 4.92 & 79.1 & 3 & $\ldots$ & $\ldots$ & $\ldots$ & ... \\
\hline J00591980-7220368 & 05919.80 & -722036.8 & 11.23 & 0.28 & 12.44 & 0.52 & 1 & 14.89 & 81.4 & 3 & F8I & ... & $\ldots$ & $\cdots$ \\
\hline J00592249-7121142 & 05922.49 & $-7121 \quad 14.3$ & 11.61 & 0.34 & 13.02 & 0.53 & 4 & -0.3 & 118.7 & 3 & & ... & $\ldots$ & $\ldots$ \\
\hline J00593268-7322538 & 05932.68 & 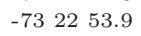 & 9.87 & 0.41 & 11.51 & & 3 & 9.33 & 72.1 & 3 & G5 & $\mathrm{G} 2.5 \mathrm{~V}$ & 12 & Flo 387 \\
\hline J00593889-7216324 & 05938.91 & $-72 \quad 1632.4$ & 10.45 & 0.62 & 12.81 & 0.97 & 1 & 9.59 & 90.9 & 3 & KoIII & $\ldots$ & $\ldots$ & $\ldots$ \\
\hline J00594007-7213368 & 05940.09 & -721336.6 & 12 & 0.18 & 12.71 & 0.18 & 1 & 126.39 & 36.8 & 1 & $\mathrm{~A} 5 \mathrm{~V}$ & & $\ldots$ & \\
\hline J00595280-7219036 & 05952.81 & -721903.6 & 12.32 & 0.03 & 12.47 & 0.08 & 1 & 169.99 & 64 & 1 & B9I & B9(Ia) & 3 & Sk $83 ;$ RMC 16 \\
\hline J00595623-7336330 & 05956.24 & -733633.1 & 10.91 & 0.63 & 13.32 & 0.85 & 4 & 61.79 & 38.3 & 3 & KOIII & & $\ldots$ & ... \\
\hline J00595831-7234188 a & 05958.34 & -723418.8 & 10.46 & 0.9 & 13.88 & 1.5 & 1 & 156.61 & 81.3 & 1 & & KOIa/Iab & 22 & \\
\hline J01000957-7233592 & 1009.60 & -723359.3 & 10.19 & 0.9 & 13.6 & 1.57 & 1 & 146.29 & 88.4 & 1 & $\ldots$ & $\ldots$ & $\ldots$ & SkKM 199 \\
\hline J01001889-7233161 & 1018.92 & -723316.1 & 11.47 & 0.27 & 12.56 & 0.37 & 1 & 26.09 & 55.1 & 3 & F2:I: & F1 & 11 & $\mathrm{AzV} 75 \mathrm{~F}$ \\
\hline J01002525-7149058 & 1025.25 & -714905.9 & 9.67 & 0.39 & 11.26 & & 3 & -10.4 & 81.9 & 3 & & G1IV & 12 & Flo 399 \\
\hline J01003301-7211289 & 1033.04 & -721128.9 & 10.52 & 0.91 & 13.77 & 1.43 & 1 & 153.39 & 98.9 & 1 & $\ldots$ & KOIb & 12 & \\
\hline
\end{tabular}


Table 3-Continued

\begin{tabular}{|c|c|c|c|c|c|c|c|c|c|c|c|c|c|c|}
\hline 2MASS & $\alpha(\mathrm{J} 2000)$ & $\delta(\mathrm{J} 2000)$ & K & $J-K$ & V & $B-V$ & Ref. $^{\mathrm{b}}$ & $\begin{array}{c}\mathrm{Vel} \\
\left(\mathrm{km} \mathrm{s}^{-1}\right)\end{array}$ & $r^{\mathrm{c}}$ & Category $^{\mathrm{d}}$ & $\begin{array}{l}\text { Hydra } \\
\text { Class }\end{array}$ & $\begin{array}{c}\text { Literature } \\
\text { Class }\end{array}$ & Ref. $^{e}$ & Comments $^{\mathrm{f}}$ \\
\hline J01004306-7321576 & 1043.08 & -732157.8 & 10.73 & 0.3 & 12.01 & 0.53 & 1 & 10.03 & 36.1 & 3 & G5 & & $\ldots$ & \\
\hline J01004445-7223551 & 1044.45 & -722355.1 & 12.85 & 0.01 & 12.73 & -0.05 & 1 & 172.49 & 38.8 & 1 & B2I & B2.5Iab & 7 & $\mathrm{AzV} 257$; $\mathrm{Sk} 91$ \\
\hline J01004635-7341475 & 1046.35 & -734147.6 & 11.27 & 0.39 & 12.82 & 0.52 & 4 & 42.09 & 34.6 & 3 & G0V & 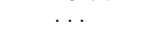 & $\ldots$ & . \\
\hline J01005063-7228521 & 1050.64 & $-72 \quad 2852.1$ & 12.62 & 0.09 & 13.1 & 0.14 & 1 & 163.74 & 38.5 & 1 & & & $\cdots$ & \\
\hline J01005338-7229023 & 1053.39 & -722902.4 & 10.07 & 0.91 & 13.41 & 1.55 & 1 & 162.29 & 83.1 & 1 & $\ldots$ & G8Iab & 12 & SkKM 206 \\
\hline J01005415-7251367 & 1054.13 & -725136.7 & 8.68 & 0.84 & 12.2 & 1.66 & 1 & 183.64 & 68.8 & 1 & & G5.5Ia & 12 & SkKM 207 \\
\hline J01005444-7416521 a & 1054.43 & -741652.1 & 11.31 & 0.41 & 12.97 & 0.5 & 5 & 6.22 & 73.3 & 3 & $\ldots$ & $\ldots$ & $\ldots$ & $\ldots$ \\
\hline J01010075-7350069 a & 1100.76 & -735007.0 & 11.29 & 0.29 & 12.69 & $\ldots$ & 3 & 16.89 & 34.1 & 3 & G0V & $\ldots$ & $\cdots$ & $\cdots$ \\
\hline J01010873-7433309 & 1108.74 & -743331.1 & 9.58 & 0.35 & 11 & $\ldots$ & 3 & -18.98 & 83.1 & 3 & $\ldots$ & F7V & 6 & Flo 421 \\
\hline J01011042-7132198 & 1110.42 & -713219.9 & 8.76 & 0.26 & 9.76 & 0.35 & 2 & 58.4 & 33.3 & 3 & $\ldots$ & A9V & 9 & RMC 10F; HD 6193 \\
\hline J01011237-7137191 & 1112.37 & -713719.1 & 10.5 & 0.66 & 12.84 & 0.97 & 1 & 21.2 & 111.7 & 3 & & $\ldots$ & $\ldots$ & ... \\
\hline J01011249-7216069 & 1112.49 & -721606.8 & 12.2 & 0.14 & 12.67 & 0.09 & 1 & 170.79 & 40.4 & 1 & $\mathrm{~A} 3 \mathrm{~V}$ & A2 2 & 11 & $\mathrm{AzV} 80 \mathrm{~F}$ \\
\hline J01011983-7217042 & $\begin{array}{lll}1 & 1 & 19.84\end{array}$ & -721704.2 & 10.6 & 0.82 & 13.76 & 1.51 & 1 & 161.09 & 89.5 & 1 & & G7Ia/Iab & 22 & \\
\hline J01012102-7130084 & 1121.04 & -713008.5 & 10.41 & 0.9 & 13.89 & 1.33 & 5 & 135.3 & 82.2 & 1 & $\ldots$ & & $\cdots$ & SkKM 215 \\
\hline J01012123-7314445 & 1121.23 & -731444.7 & 10.3 & 0.24 & 11.48 & & 3 & 20.49 & 48.8 & 3 & & F7V & 6 & Flo 422 \\
\hline J01012215-7202033 & 1122.15 & -720203.2 & 9.03 & 0.86 & 11.93 & 1.24 & 1 & -32.39 & 103.2 & 3 & KoIII & K2III & 12 & \\
\hline J01012272-7235404 & 1122.73 & -723540.4 & 11.42 & 0.3 & 12.77 & 0.56 & 1 & 7.29 & 70 & 3 & F8 & $\ldots$ & $\cdots$ & $\cdots$ \\
\hline J01012743-7207062 a & 1127.43 & -720706.1 & 11.78 & 0.07 & 12.17 & 0.06 & 2 & 108.45 & 55.2 & 1 & A0I & A0 & 13 & $\mathrm{AzV} 273 ; \mathrm{Sk} 99$ \\
\hline J01014157-7315244 & 1141.57 & $-73 \quad 15 \quad 24.5$ & 11.72 & 0.35 & 13.12 & 0.6 & 1 & 6.69 & 35.5 & 3 & & & $\cdots$ & \\
\hline J01014382-7218025 & 1143.83 & -721802.6 & 11.36 & 0.37 & 12.81 & 0.61 & 1 & 44.99 & 85.3 & 3 & G2III & $\ldots$ & $\ldots$ & $\ldots$ \\
\hline J01014559-7223223 & 1145.59 & -722322.3 & 10.95 & 0.57 & 13.21 & 0.96 & 1 & 13.09 & 84.4 & 3 & & & . & N \\
\hline J01014948-7205454 a & 1149.49 & -720545.3 & 10.79 & 0.63 & 13.15 & 0.96 & 1 & 177.85 & 82.6 & 1 & $\mathrm{G} 2$ & G1Iab & 12 & N \\
\hline $\mathrm{J} 01015742-7204172 \mathrm{a}$ & 1157.43 & $-7204 \quad 17.1$ & 11.58 & 0.17 & 12.25 & 0.15 & 1 & 123.65 & 42 & 1 & A5I & A3I & 13 & Sk $102 ; \mathrm{AzV} 286 ; \mathrm{RMC} \mid 22$ \\
\hline J01020100-7122208 & 1201.01 & -712220.8 & 10.81 & 0.78 & 13.75 & 1.15 & 4 & 296.1 & 61.3 & 1 & & & $\ldots$ & \\
\hline J01020466-7431060 & 1204.68 & $\begin{array}{lll}-74 & 31 & 06.1\end{array}$ & 9.36 & 0.84 & 12.33 & 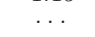 & 3 & 91.35 & 122.4 & 2 & $\ldots$ & $\ldots$ & $\ldots$ & $\ldots$ \\
\hline J01020591-7358015 & 1205.91 & -735801.7 & 10.97 & 0.59 & 13.31 & 0.85 & 5 & 84.1 & 97.7 & 3 & & $\cdots$ & $\cdots$ & $\cdots$ \\
\hline J01020977-7155354 & 1209.78 & -715535.5 & 9.85 & 0.75 & 12.64 & 1.14 & 1 & 117.41 & 88.3 & 2 & G5I & $\ldots$ & $\ldots$ & $\ldots$ \\
\hline J01020979-7200232 a & 1209.80 & -720023.2 & 12.21 & 0 & 12.1 & -0.03 & 1 & 169.45 & 56.2 & 1 & B5I & B8Ia & 7 & $\mathrm{AzV} 297 ; \mathrm{RMC} 23$ \\
\hline J01021027-7348571 & 1210.27 & -734857.3 & 10.53 & 0.42 & 12.25 & & 3 & 4.59 & 60.2 & 3 & $\mathrm{G} 5 \mathrm{~V}$ & & $\ldots$ & \\
\hline J01021230-7202516 a & 1212.32 & -720251.5 & 12.33 & 0.02 & 12.47 & 0.03 & 1 & 154.85 & 49.9 & 1 & AOI & AOI & 14 & $\mathrm{AzV} 298 ; \mathrm{RMC} 24$ \\
\hline J01021987-7145570 & 1219.87 & -714557.0 & 11.28 & 0.42 & 12.93 & 0.6 & 1 & 12.51 & 59.5 & 3 & $\mathrm{G} 2 \mathrm{~V}$ & & $\ldots$ & \\
\hline J01022044-7415374 a & 1220.43 & -741537.6 & 9.46 & 0.7 & 12.05 & 1.02 & 5 & 19.82 & 103.7 & 3 & & & ... & \\
\hline J01022196-7404492 & 1221.97 & -740449.3 & 10.66 & 0.35 & 11.92 & & 3 & -13.05 & 80.4 & 3 & & F2: & 6 & Flo 441 \\
\hline J01022209-7146368 a & $\begin{array}{ll}1222.09 \\
\end{array}$ & -714636.9 & 11.59 & 0.29 & 12.74 & 0.43 & 1 & -13.8 & 64.7 & 3 & F2II & & $\ldots$ & $\ldots$ \\
\hline J01022649-7202284 & 1226.50 & -720228.4 & 9.84 & 0.36 & 11.29 & $\ldots$ & 3 & 16.39 & 83.1 & 3 & F8V & GOV & 12 & Flo 438 \\
\hline J01022712-7216454 & $\begin{array}{ll}1227.13 \\
\end{array}$ & -721645.5 & 11.88 & 0.22 & 12.74 & 0.24 & 1 & 131.59 & 36.7 & 1 & A3I & F5I & 8 & Flo $439 ; \mathrm{AzV} 305$ \\
\hline J01023088-7223578 & 1230.90 & -722357.8 & 8.71 & 0.64 & 11.07 & 1.02 & 1 & 2.89 & 131.1 & 3 & KOIII & G8II & 12 & Flo 443 \\
\hline J01023575-7149423 & 1235.77 & -714942.4 & 9.98 & 0.91 & 13.52 & 1.57 & 1 & 186.91 & 88.5 & 1 & $\mathrm{~K} 2 \mathrm{I}$ & & $\ldots$ & SkKM 233 \\
\hline J01023730-7216250 & 1237.31 & -721625.0 & 8.97 & 0.9 & 12.55 & 1.74 & 1 & 158.29 & 82.2 & 1 & & G6Ia/Iab & 22 & SkKM 235 \\
\hline J01023810-7208066 & 1238.12 & -720806.6 & 12.4 & 0.1 & 12.72 & 0.06 & 1 & 135.39 & 45.6 & 1 & A3I & & & \\
\hline J01023991-7211273 & 1239.92 & -721127.3 & 10.15 & 0.81 & 13.23 & 1.25 & 1 & 127.55 & 78.5 & 1 & G8III & G7Iab/Ib & 22 & SkKM 236 \\
\hline J01024303-7207260 & 1243.04 & -720726.0 & 10.58 & 0.26 & 11.64 & 0.3 & 2 & 138.09 & 35.4 & 1 & FOI & FOIa & 10 & $\mathrm{Sk} 105 ; \mathrm{AzV} 310 ; \mathrm{RMC} 26$ \\
\hline J01024960-7210145 & 1249.60 & -721014.5 & 10.55 & 0.11 & 10.9 & 0.08 & 2 & 145.01 & 53.5 & 1 & AOI & A0Ia & 7 & CPD-72 77; Sk 106; RMC 27 \\
\hline J01025349-7213145 a & 1253.49 & -721314.5 & 8.9 & 0.65 & 11.25 & 0.97 & 1 & 24.95 & 126.6 & 3 & KOIII & G7.5III & $\cdots$ & \\
\hline J01025533-7206488 & 1255.35 & -720648.8 & 11.4 & 0.28 & 12.64 & 0.51 & 1 & 13.89 & 75.2 & 3 & F8V & & $\ldots$ & \\
\hline J01025992-7232455 & 1259.92 & -723245.5 & 7.61 & 0.69 & 10.22 & $\ldots$ & 3 & 1.89 & 118.5 & 3 & KOIII & K1IV & 12 & Flo 454 ; HD 6407 \\
\hline J01030213-7205170 & 1302.12 & -720517.0 & 8.41 & 0.29 & 9.63 & 0.46 & 2 & 36.51 & 92.8 & 3 & F5III & F9III & 12 & RMC 11F; HD 6406 \\
\hline J01030464-7306289 & 1304.66 & -730629.1 & 7.28 & 0.91 & 10.6 & & 3 & -57.11 & 105.8 & 3 & & $\mathrm{~K} 2 \mathrm{~V}$ & 6 & Flo 455 \\
\hline J01030765-7212362 & 1307.67 & -721236.3 & 12.45 & 0.09 & 12.95 & 0.13 & 1 & 156.19 & 41.5 & 1 & FoIb & A3 & 11 & $\mathrm{AzV} 90 \mathrm{~F}$ \\
\hline J01030892-7155507 & 1308.92 & -715550.8 & 9.55 & 0.88 & 13.12 & 1.75 & 1 & 141.31 & 75.8 & 1 & G5: & KOIab & 12 & SkKM 242 \\
\hline
\end{tabular}


Table 3-Continued

\begin{tabular}{|c|c|c|c|c|c|c|c|c|c|c|c|c|c|c|}
\hline 2MASS & $\alpha(\mathrm{J} 2000)$ & $\delta(\mathrm{J} 2000)$ & K & $J-K$ & V & $B-V$ & Ref. ${ }^{\text {b }}$ & $\begin{array}{c}\mathrm{Vel} \\
\left(\mathrm{km} \mathrm{s}^{-1}\right)\end{array}$ & $r^{\mathrm{c}}$ & Category $^{\mathrm{d}}$ & $\begin{array}{c}\text { Hydra } \\
\text { Class }\end{array}$ & $\begin{array}{c}\text { Literature } \\
\text { Class }\end{array}$ & Ref. $^{\mathrm{e}}$ & Comments $^{f}$ \\
\hline J01031418-7221588 & 1314.16 & -722158.9 & 7.35 & 0.63 & 9.74 & 1.03 & 2 & 3.99 & 117.8 & 3 & KOIII & G7IV & 12 & Flo 457; HD 6431 \\
\hline J01032108-7204478 & 1321.08 & $-7204 \quad 47.9$ & 11.31 & 0.26 & 12.46 & 0.44 & 1 & 5.89 & 86.4 & 3 & F8III & & & \\
\hline J01032294-7236126 & 1322.94 & $\begin{array}{lll}-72 & 36 & 12.7\end{array}$ & 10.23 & 0.89 & 13.67 & 1.58 & 1 & 165.09 & 61.5 & 1 & G2? & KOIab/Ib & 22 & SkKM 251 \\
\hline J01033277-7224565 & 1332.78 & -722456.7 & 10.6 & 0.78 & 13.68 & 1.44 & 1 & 153.29 & 80.9 & 1 & & G5.5Iab & 12 & SkKM 254 \\
\hline J01034304-7215299 a & 1343.04 & -721530.0 & 12.43 & 0.04 & 12.54 & 0.03 & 1 & 158.85 & 50.1 & 1 & $\mathrm{~A} 0 \mathrm{I}$ & A0I & 14 & $\mathrm{AzV}$ 338; Sk 110 \\
\hline J01034677-7209106 & 1346.78 & -720910.8 & 10.88 & 0.41 & 12.42 & 0.64 & 1 & -13.09 & 103.1 & 3 & G5III & & & $\ldots$ \\
\hline J01034794-7338348 & 1347.96 & -733835.1 & 9.69 & 0.7 & 12.31 & & 3 & 35.45 & 78.2 & 3 & & KoIII & 12 & $\ldots$ \\
\hline J01034909-7342442 & 1349.09 & -734244.6 & 10.68 & 0.78 & 13.61 & 1.47 & 4 & 194.85 & 49.1 & 1 & .. & G4.5Iab & 12 & \\
\hline J01034942-7207296 & 1349.45 & $\begin{array}{lll}-72 & 07 & 29.7\end{array}$ & 12.82 & 0 & 12.71 & -0.06 & 1 & 181.21 & 24.9 & 1 & B2I & B1Ia & 7 & $\mathrm{AzV} 340 ; \mathrm{RMC} 33$ \\
\hline J01035385-7423020 & 1353.83 & $\begin{array}{llll}-74 & 23 & 02.1\end{array}$ & 11.58 & 0.37 & 13.01 & 0.72 & 5 & -7.35 & 81.8 & 3 & & K0.5III & 12 & \\
\hline J01035882-7327447 a & 1358.83 & -732744.8 & 5.51 & 0.91 & 8.7 & $\ldots$ & 3 & 4.27 & 98.1 & 3 & $\cdots$ & $\mathrm{K} 2$ & 9 & Flo 469; HD 6509 \\
\hline J01035883-7223090 & 1358.84 & $\begin{array}{lll}-72 & 23 & 09.0\end{array}$ & 10.59 & 0.7 & 13.35 & 1.26 & 1 & 139.59 & 60.6 & 1 & $\ldots$ & & & SkKM 263 \\
\hline J01040165-7208254 & 1401.67 & -720825.3 & 7.83 & 0.87 & 11.14 & 1.42 & 1 & -0.21 & 105.9 & 3 & K5III & K4III/IV & 22 & $\ldots$ \\
\hline J01040239-7412520 & 1402.39 & $\begin{array}{lll}-74 & 12 & 51.9\end{array}$ & 10.45 & 0.59 & 12.72 & 0.88 & 5 & 106.75 & 79.2 & 2 & $\ldots$ & $\ldots$ & $\ldots$ & \\
\hline J01040596-7349058 & 1405.96 & -734905.8 & 12.02 & 0.21 & 13.08 & & 3 & 218.55 & 29.1 & 1 & . & Fo & 3 & $\mathrm{AzV} 99 \mathrm{~F}$ \\
\hline J01041045-7154025 & 1410.46 & -715402.5 & 11.31 & 0.4 & 12.82 & 0.69 & 1 & 61.31 & 120.7 & 3 & $\mathrm{G} 2$ & & $\ldots$ & $\ldots$ \\
\hline J01041108-7308494 & 1411.08 & $-7308 \quad 49.4$ & 10.73 & 0.35 & 12.21 & 0.66 & 1 & 37.69 & 105.2 & 3 & & ... & $\ldots$ & $\ldots$ \\
\hline J01041148-7143516 a & 1411.48 & -714351.6 & 10.28 & 0.58 & 12.61 & 1 & 1 & 11.3 & 76.4 & 3 & G8III & & & \\
\hline J01041227-7152032 & 1412.28 & $\begin{array}{llll}-71 & 52 & 03.2\end{array}$ & 11.9 & 0.02 & 12.1 & -0.01 & 2 & 146.71 & 47.8 & 1 & AOI & A0I & 13 & $\mathrm{AzV} 347$; Sk 113 \\
\hline J01041450-7311470 & 1414.50 & $\begin{array}{llll}-73 & 11 & 47.1\end{array}$ & 11.24 & 0.55 & 13.38 & 0.81 & 1 & 4.89 & 48.1 & 3 & & $\ldots$ & $\cdots$ & $\ldots$ \\
\hline J01041658-7224012 & 1416.58 & $\begin{array}{lll}-72 & 24 & 01.2\end{array}$ & 9.36 & 0.28 & 10.49 & 0.44 & 2 & 7.39 & 83.5 & 3 & F2I & $\mathrm{F} 2$ & 6 & Flo 474 ; HD 6537 \\
\hline J01041885-7203431 & 1418.86 & -720343.1 & 11.43 & 0.46 & 13.21 & 0.67 & 1 & 8.95 & 70.3 & 3 & $\mathrm{G} 5 \mathrm{~V}$ & & & $\ldots$ \\
\hline J01042100-7215096 a & 1420.98 & $\begin{array}{llll}-72 & 15 & 09.7\end{array}$ & 6.1 & 0.6 & 8.42 & 1 & 2 & 1.25 & 129.6 & 3 & G8 & G8III & 12 & RMC 16F; HD 6536 \\
\hline J01042391-7311565 & 1423.90 & -731156.6 & 8.92 & 0.27 & 9.92 & $\ldots$ & 3 & -10.81 & 106.4 & 3 & $\ldots$ & F9IV/V & 12 & Flo 481; HD 6561 \\
\hline J01042527-7231188 & 1425.27 & $-7231 \quad 18.8$ & 10.1 & 0.65 & 12.57 & 1 & 1 & 42.19 & 80.9 & 3 & G5V & K0.5III & 12 & $\ldots$ \\
\hline J01042815-7408183 & 1428.14 & $\begin{array}{lll}-74 & 08 & 18.3\end{array}$ & 11.34 & 0.42 & 13.07 & $\cdots$ & 3 & 26.45 & 99 & 3 & $\ldots$ & & & $\cdots$ \\
\hline J01043056-7130447 & 1430.56 & -713044.8 & 9.48 & 0.63 & 12.03 & $\ldots$ & 3 & -23 & 83.2 & 3 & $\ldots$ & $\ldots$ & $\ldots$ & $\ldots$ \\
\hline J01043573-7204155 & 1435.74 & $-7204 \quad 15.5$ & 10.54 & 0.8 & 13.65 & 1.47 & 1 & 154.89 & 97.8 & 1 & & & & \\
\hline J01044935-7206218 a & 1449.35 & -720621.8 & 11.34 & 0.03 & 11.36 & -0.02 & 2 & 189.85 & 50.3 & 1 & B1II & B3Ia & 7 & Sk 114; AzV 362; RMC 36 \\
\hline J01045290-7208368 & 1452.91 & -720836.8 & 10.88 & 0.13 & 11.23 & 0.07 & 2 & 194.01 & 67.8 & 1 & B9I & B9Ia+ & 7 & Sk 117; AzV 367; RMC 37 \\
\hline J01045356-7419475 & 1453.54 & -741947.6 & 10.72 & 0.33 & 12.13 & 0.39 & 4 & -47.15 & 79.9 & 3 & - & F6V & 6 & Flo 489 \\
\hline J01045902-7208503 & 1459.04 & $-7208 \quad 50.2$ & 8.09 & 0.72 & 11 & 1.04 & 4 & 2.59 & 99.2 & 3 & K2III & $\ldots$ & $\ldots$ & K1IIIe \\
\hline J01045990-7126108 & 1459.90 & $\begin{array}{lll}-71 & 26 & 10.9\end{array}$ & 11.63 & 0.34 & 13.18 & 0.67 & 4 & 68.5 & 89.4 & 3 & $\cdots$ & & & $\ldots$ \\
\hline J01051187-7143346 & 1511.87 & $-7143 \quad 34.6$ & 10.63 & 0.84 & 13.73 & 1.5 & 1 & 144.61 & 82.7 & 1 & $\ldots$ & $\ldots$ & $\ldots$ & SkKM 283 \\
\hline J01051190-7417474 & 1511.89 & $\begin{array}{llll}-74 & 17 & 47.4\end{array}$ & 10.46 & 0.61 & 12.86 & 1.03 & 4 & -33.25 & 129.5 & 3 & .. & $\ldots$ & $\ldots$ & ... \\
\hline J01051279-7159337 & 1512.81 & -715933.8 & 10.43 & 0.91 & 13.8 & 1.31 & 1 & 159.41 & 88.3 & 1 & K5? & $\ldots$ & $\ldots$ & \\
\hline J01052460-7255293 & 1524.59 & $\begin{array}{lll}-72 & 55 & 29.4\end{array}$ & 10.08 & 0.87 & 13.25 & 1.4 & 1 & 161.39 & 53.5 & 1 & G5?I? & .. & $\cdots$ & SkKM 285; G8Iab \\
\hline J01052811-7127504 & 1528.13 & -712750.5 & 10.27 & 0.41 & 12.01 & 0.74 & 4 & -0.3 & 125.9 & 3 & & .. & $\cdots$ & \\
\hline J01054102-7221246 & 1541.04 & -722124.6 & 10.43 & 0.91 & 13.71 & 1.53 & 1 & 191.59 & 70.2 & 1 & G2:III: & & $\ldots$ & SkKM 290; G8Ia/Iab \\
\hline J01055631-7219448 a & 1556.32 & -721944.8 & 11.03 & 0.15 & 11.47 & -0.03 & 2 & 137.85 & 39 & 1 & $\mathrm{~B} 2 \mathrm{I}(\mathrm{e})$ & B2Ia & 16 & Sk 124; AzV 393; RMC 39 \\
\hline J01060182-7217252 & 1601.85 & $\begin{array}{lll}-72 & 17 & 25.2\end{array}$ & 9.19 & 0.57 & 11.35 & 0.89 & 1 & -12.49 & 120.2 & 3 & G8III & $\ldots$ & $\cdots$ & G8IV \\
\hline J01065126-7255533 & 1651.28 & -725553.4 & 9.06 & 0.69 & 11.55 & 1.08 & 1 & 38.09 & 80.9 & 3 & $\ldots$ & & & KOIII \\
\hline J01065315-7357550 a & 1653.16 & -735755.2 & 11.03 & 0.28 & 12.34 & 0.33 & 4 & 23.88 & 63.4 & 3 & & & & \\
\hline J01070175-7310374 & 1701.76 & -731037.5 & 10.79 & 0.76 & 13.75 & 1.38 & 1 & 170.09 & 65.4 & 1 & $\ldots$ & $\ldots$ & $\cdots$ & SkKM 300 \\
\hline J01070284-7148269 & 1702.84 & $\begin{array}{lll}-71 & 48 & 27.1\end{array}$ & 8.41 & 0.47 & 10.39 & 0.84 & 2 & -9.39 & 129.5 & 3 & KoIII & $\mathrm{G} 8 \mathrm{~V}$ & 6 & HD 6827 \\
\hline J01070665-7346504 & 1706.66 & -734650.5 & 9.79 & 0.6 & 12.1 & . & 3 & -31.75 & 109.8 & 3 & & & $\cdots$ & \\
\hline J01070716-7336249 & 1707.17 & -733624.9 & 9.56 & 0.61 & 11.92 & $\cdots$ & 3 & 53.65 & 84.6 & 3 & . & . & $\cdots$ & $\mathrm{K} 1 \mathrm{~V}$ \\
\hline J01071373-7400250 & 1713.74 & -740025.0 & 8.51 & 0.65 & 11 & $\ldots$ & 3 & 37.62 & 83.1 & 3 & $\ldots$ & $\ldots$ & $\ldots$ & $\ldots$ \\
\hline J01071439-7401353 & 1714.39 & -740135.3 & 7.71 & 0.84 & 10.86 & $\ldots$ & 3 & -35.38 & 94.8 & 3 & $\cdots$ & $\ldots$ & $\cdots$ & $\cdots$ \\
\hline J01071905-7351075 a & 1719.06 & $\begin{array}{llll}-73 & 51 & 07.6\end{array}$ & 10.15 & 0.64 & 12.64 & 0.98 & 4 & 12.78 & 78.8 & 3 & $\ldots$ & & & $\ldots$ \\
\hline
\end{tabular}


Table 3-Continued

\begin{tabular}{|c|c|c|c|c|c|c|c|c|c|c|c|c|c|c|}
\hline 2MASS & $\alpha(\mathrm{J} 2000)$ & $\delta(\mathrm{J} 2000)$ & K & $J-K$ & V & $B-V$ & Ref. ${ }^{\text {b }}$ & $\begin{array}{c}\mathrm{Vel} \\
\left(\mathrm{km} \mathrm{s}^{-1}\right)\end{array}$ & $r^{\mathrm{c}}$ & Category $^{\mathrm{d}}$ & $\begin{array}{c}\text { Hydra } \\
\text { Class }\end{array}$ & $\begin{array}{l}\text { Literature } \\
\text { Class }\end{array}$ & Ref. $^{\text {e }}$ & Comments $^{\mathrm{f}}$ \\
\hline J01073340-7345474 & 1733.40 & -734547.6 & 10.15 & 0.36 & 11.72 & & 3 & -3.48 & 148.5 & 3 & & & ... & $\ldots$ \\
\hline J01073493-7324304 & 1734.92 & -732430.4 & 6.99 & 0.76 & 9.82 & & 3 & 15.09 & 89.7 & 3 & & K2 & 6 & Flo 536; HD 6939; K2III \\
\hline J01073584-7213426 & 1735.85 & -721342.6 & 7.15 & 0.88 & 10.47 & $\ldots$ & 3 & -0.49 & 96.9 & 3 & K3III & K5 & 6 & Flo $531 ; \mathrm{K} 4 \mathrm{III} / \mathrm{IV}$ \\
\hline J01073986-7205208 a & 1739.86 & -720520.9 & 11.44 & 0.33 & 12.93 & 0.57 & 1 & 8.78 & 71 & 3 & $\mathrm{~F} 8 \mathrm{~V}$ & & ... & \\
\hline J01074309-7212153 & 1743.10 & -721215.3 & 8.72 & 0.83 & 11.69 & 1.21 & 1 & -60.84 & 109.2 & 3 & & $\mathrm{~K} 5 \mathrm{~V}$ & 2 & SMC 66510; K2III \\
\hline J01074659-7203564 & 1746.60 & -720356.5 & 10.66 & 0.19 & 11.62 & & 3 & 18.11 & 57.9 & 3 & F2III & & & \\
\hline J01080317-7149496 a & 1803.18 & -714949.7 & 10.35 & 0.57 & 12.6 & 0.94 & 1 & 45.08 & 77.5 & 3 & & & ... & $\cdots$ \\
\hline J01080549-7208426 & 1805.50 & -720842.8 & 12.41 & 0.11 & 12.95 & 0.12 & 1 & 160.81 & 30.4 & 1 & A2I & A2I & 8 & $\mathrm{AzV} 431 ; \mathrm{Sk} 134$ \\
\hline J01080736-7148502 a & 1807.36 & -714850.3 & 10.39 & 0.84 & 13.63 & 1.55 & 1 & 159.78 & 73.6 & 1 & $\ldots$ & & ( & SkKM 307 \\
\hline J01081243-7141278 & 1812.44 & -714127.9 & 11.44 & 0.31 & 12.64 & 0.38 & 1 & 176.86 & 30.5 & 1 & $\ldots$ & F5I & 8 & $\mathrm{AzV} 434$ \\
\hline J01083857-7150069 a & 1838.58 & -715007.0 & 6.43 & 0.55 & 8.57 & $\ldots$ & 3 & -8.82 & 124.2 & 3 & G0I & hG5gG5mF6 & 19 & HD 7031 \\
\hline J01084312-7207344 & 1843.13 & $\begin{array}{llll}-72 & 07 & 34.4\end{array}$ & 9.68 & 0.52 & 11.72 & 0.87 & 1 & 12.11 & 87.7 & 3 & KOIII & K1 & 6 & Flo 552; K3V \\
\hline J01084882-7316027 & 1848.83 & -731602.8 & 10.87 & 0.65 & 13.36 & 1.07 & 1 & -31.11 & 94.5 & 3 & & & $\ldots$ & 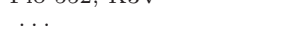 \\
\hline J01085451-7208214 a & 1854.53 & -720821.4 & 10.16 & 0.91 & 13.58 & 1.55 & 1 & 128.08 & 82.3 & 1 & $\ldots$ & & .. & SkKM 313 \\
\hline J01085685-7302344 & 1856.85 & $\begin{array}{llll}-73 & 02 & 34.4\end{array}$ & 10.78 & 0.14 & 11.35 & 0.13 & 2 & 178.79 & 50.8 & 1 & $\ldots$ & A3Ia & 10 & Sk 136; AzV 442; RMC 43 \\
\hline J01091296-7401274 & 1912.93 & -740127.4 & 9.59 & 0.68 & 12.17 & 0.87 & 4 & 19.92 & 85 & 3 & & & & \\
\hline J01091733-7151168 a & 1917.33 & $-7151 \quad 16.8$ & 8.7 & 0.65 & 11.17 & $\ldots$ & 3 & 14.88 & 95.3 & 3 & K1III & G8 & 6 & Flo 563 \\
\hline J01093700-7318030 & 1937.01 & -731803.1 & 12.77 & 0.04 & 13.08 & 0.08 & 1 & 159.99 & 34.2 & 1 & $\ldots$ & A2II & 3 & $2 \mathrm{dFS} 2579 ; \mathrm{AzV} 115 \mathrm{~F}$ \\
\hline J01100842-7349103 & 11008.40 & -734910.5 & 11.16 & 0.29 & 12.4 & 0.45 & 4 & -13.68 & 39.3 & 3 & & & $\cdots$ & $\ldots$ \\
\hline J01101248-7237310 & 11012.48 & -723731.0 & 10.11 & 0.87 & 13.56 & 1.58 & 1 & 196.01 & 67.7 & 1 & $\ldots$ & $\ldots$ & $\ldots$ & SkKM 320; G8.5Ia/Iab \\
\hline J01101910-7233515 & 11019.10 & -723351.5 & 11.47 & 0.41 & 13.19 & 0.8 & 1 & 10.81 & 29.4 & 3 & G8V & $\ldots$ & $\ldots$ & N \\
\hline J01102381-7157353 & 11023.79 & -715735.3 & 10.51 & 0.91 & 13.85 & 1.57 & 1 & 192.86 & 64.9 & 1 & & . & $\ldots$ & SkKM 321 \\
\hline J01104014-7228304 & 11040.14 & -722830.4 & 11.18 & 0.48 & 13.05 & 0.77 & 1 & 4.61 & 62.5 & 3 & G5 & $\cdots$ & $\cdots$ & $\ldots$ \\
\hline J01104331-7148491 & 11043.30 & -714849.1 & 9.21 & 0.59 & 11.45 & & 3 & 8.76 & 95 & 3 & & G8 & 6 & Flo 588 \\
\hline J01105850-7248346 & 11058.48 & -724834.6 & 9.78 & 0.82 & 12.8 & 1.34 & 1 & 33.71 & 37 & 3 & K3:IIIe & $\ldots$ & $\ldots$ & K3.5III \\
\hline J01110839-7143116 & 11108.39 & -714311.5 & 10.72 & 0.54 & 12.7 & 0.81 & 1 & -24.24 & 56.8 & 3 & & & $\cdots$ & $\ldots$ \\
\hline J01110988-7306296 & 11109.87 & -730629.7 & 11.21 & 0.56 & 13.45 & 1 & 1 & 1.34 & 57.1 & 3 & KoIII & $\ldots$ & & $\ldots$ \\
\hline J01112045-7222169 & 11120.44 & -722216.9 & 10.06 & 0.26 & 11.25 & $\ldots$ & 3 & 14.41 & 58.7 & 3 & $\ldots$ & $\mathrm{F} 2$ & 6 & Flo 598 \\
\hline J01112540-7317042 & 11125.39 & -731704.3 & 10.29 & 0.88 & 13.61 & 1.4 & 1 & 162.84 & 81.6 & 1 & G2I & 10 & $\ldots$ & $\ldots$ \\
\hline J01112782-7258501 & 11127.81 & -725850.2 & 10.57 & 0.4 & 12.13 & 0.65 & 1 & -13.36 & 74.3 & 3 & $\mathrm{G} 2 \mathrm{~V}$ & .. & $\ldots$ & $\ldots$ \\
\hline J01114315-7207275 & 11143.17 & -720727.5 & 11.64 & 0.13 & 12.1 & 0.09 & 1 & 151.36 & 23.1 & 1 & & A2I & 13 & $\mathrm{AzV}$ 463; Sk 146 \\
\hline J01115007-7202309 & 11150.08 & -720231.0 & 11.19 & 0.5 & 13.27 & 0.86 & 1 & 32.66 & 48.3 & 3 & $\cdots$ & & & \\
\hline J01115008-7216215 a & 11150.07 & -721621.6 & 8.17 & 0.62 & 10.57 & & 3 & 0.83 & 124.9 & 3 & $\cdots$ & G5III? & 6 & Flo 608 ; CD-72 55 \\
\hline J01115339-7157036 & 11153.39 & -715703.6 & 11.52 & 0.28 & 12.79 & 0.47 & 1 & -6.74 & 45 & 3 & $\ldots$ & $\ldots$ & & ... \\
\hline J01115681-7202186 & 11156.84 & -720218.7 & 11.08 & 0.61 & 13.51 & 0.99 & 1 & 37.86 & 52.3 & 3 & $\ldots$ & $\ldots$ & $\ldots$ & $\ldots$ \\
\hline J01121070-7337312 & 11210.69 & $\begin{array}{lll}-73 & 37 & 31.2\end{array}$ & 8.29 & 0.74 & 11.06 & 1.24 & 1 & 52.52 & 92.7 & 3 & & K6: & 6 & Flo 616 \\
\hline J01123076-7259262 & 11230.76 & $\begin{array}{lll}-72 & 59 & 26.4\end{array}$ & 10.15 & 0.78 & 13.01 & 1.12 & 1 & -10.36 & 107.2 & 3 & KOIII & $\ldots$ & & \\
\hline J01123517-7309353 & 11235.17 & -730935.5 & 10.6 & 0.85 & 13.85 & 1.47 & 1 & 173.74 & 78 & 1 & $\ldots$ & $\ldots$ & & SkKM 327 \\
\hline J01124028-7352208 & 11240.29 & $-7352 \quad 21.0$ & 8.86 & 0.79 & 11.65 & 1.1 & 1 & 106.52 & 85.3 & 2 & $\ldots$ & & $\ldots$ & \\
\hline J01130112-7358126 & $\begin{array}{lll}1 & 13 & 01.14\end{array}$ & $\begin{array}{lll}-73 & 58 & 12.8\end{array}$ & 10.2 & 0.36 & 11.53 & & 3 & 28.12 & 86.7 & 3 & $\ldots$ & F7 & 6 & Flo 630 \\
\hline J01130227-7241420 & 11302.27 & -724142.1 & 10.91 & 0.49 & 12.82 & 0.83 & 1 & -3.89 & 39.9 & 3 & & & ... & \\
\hline J01130838-7320236 & 11308.40 & -732023.8 & 11.2 & 0.42 & 12.81 & 0.61 & 1 & 188.94 & 46.6 & 1 & F8I & F5I? & 8 & $\mathrm{AzV} 473$ \\
\hline J01131654-7208516 & 11316.56 & -720851.6 & 10.49 & 0.4 & 12.2 & 0.72 & 1 & 17.86 & 80.3 & 3 & & & & \\
\hline J01132351-7328318 & 11323.52 & $\begin{array}{lll}-73 & 28 & 31.9\end{array}$ & 9.3 & 0.38 & 10.87 & $\ldots$ & 3 & 1.24 & 121.9 & 3 & $\mathrm{G} 2 \mathrm{~V}$ & G1V & 6 & Flo 639 \\
\hline J01132650-7400500 & 11326.51 & -740050.1 & 9.78 & 0.59 & 11.97 & 0.87 & 1 & 38.12 & 74.3 & 3 & $\ldots$ & $\ldots$ & $\ldots$ & $\ldots$ \\
\hline J01133207-7239069 & 11332.08 & -723906.9 & 10.99 & 0.28 & 12.21 & 0.52 & 1 & 26.21 & 78.8 & 3 & & & $\ldots$ & $\ldots$ \\
\hline J01133660-7255139 & 11336.63 & -725514.0 & 10.34 & 0.86 & 13.39 & 1.44 & 1 & 173.33 & 81.7 & 1 & $\mathrm{G} 2 \mathrm{~V}$ & $\ldots$ & $\ldots$ & SkKM 329 \\
\hline J01135159-7325262 & 11351.60 & $\begin{array}{lll}-73 & 25 & 26.4\end{array}$ & 8.53 & 0.64 & 10.9 & & 3 & -42.36 & 114.2 & 3 & $\mathrm{~K} 1 \mathrm{~V}$ & G9 & 6 & Flo 650 \\
\hline J01135200-7327054 & 11352.01 & -732705.6 & 12.45 & 0.12 & 13.16 & 0.29 & 1 & 18.04 & 33.9 & 3 & A5-F2 & & $\ldots$ & $\ldots$ \\
\hline J01135403-7323500 & 11354.03 & $\begin{array}{lll}-73 & 23 & 50.2\end{array}$ & 11.74 & 0.34 & 13.09 & 0.46 & 1 & 192.94 & 32.3 & 1 & FOI & . & $\ldots$ & $\ldots$ \\
\hline
\end{tabular}


Table 3-Continued

\begin{tabular}{|c|c|c|c|c|c|c|c|c|c|c|c|c|c|c|}
\hline 2MASS & $\alpha(\mathrm{J} 2000)$ & $\delta(\mathrm{J} 2000)$ & $K$ & $J-K$ & V & $B-V$ & Ref. $^{\text {b }}$ & $\begin{array}{c}\mathrm{Vel} \\
\left(\mathrm{km} \mathrm{s}^{-1}\right)\end{array}$ & $r^{\mathrm{c}}$ & Category $^{\mathrm{d}}$ & $\begin{array}{c}\text { Hydra } \\
\text { Class }\end{array}$ & $\begin{array}{c}\text { Literature } \\
\text { Class }\end{array}$ & Ref. ${ }^{\mathrm{e}}$ & Comments ${ }^{f}$ \\
\hline J01135405-7251396 & 11354.06 & -725139.6 & 9.84 & 0.89 & 13.18 & 1.54 & 1 & 132.84 & 84 & 1 & G0I: & $\ldots$ & $\ldots$ & SkKM 330 \\
\hline J01135944-7402377 & 11359.43 & -740237.8 & 10.28 & 0.62 & 12.61 & 0.93 & 1 & -1.18 & 60.4 & 3 & $\ldots$ & $\ldots$ & $\ldots$ & \\
\hline J01141827-7342084 & 11418.28 & -734208.5 & 10.8 & 0.29 & 12.04 & 0.48 & 1 & 27.42 & 89.2 & 3 & . & F7 & 6 & Flo 657 \\
\hline J01142725-7242297 & 11427.27 & -724229.8 & 11.16 & 0.36 & 12.64 & 0.69 & 1 & 25.91 & 37.1 & 3 & $\ldots$ & $\ldots$ & $\ldots$ & $\ldots$ \\
\hline J01142805-7239535 & 11428.06 & -723953.5 & 11.22 & 0.55 & 13.04 & 0.98 & 1 & 173.91 & 48.7 & 1 & $\ldots$ & F5Ib & 15 & HV 2195; RMC 46 \\
\hline J01143413-7302354 & 11434.14 & -730235.5 & 11.05 & 0.62 & 13.46 & 1.01 & 1 & 12.84 & 77.6 & 3 & KOIII & $\ldots$ & $\ldots$ & ... \\
\hline J01144168-7219101 & 11441.69 & -721910.1 & 11.45 & 0.41 & 13.04 & 0.73 & 1 & 23.41 & 28 & 3 & & $\ldots$ & $\ldots$ & $\ldots$ \\
\hline J01144335-7309572 & 11443.36 & -730957.3 & 10.79 & 0.33 & 12.16 & 0.54 & 1 & 11.74 & 73.9 & 3 & F8 V & $\ldots$ & $\ldots$ & $\ldots$ \\
\hline J01144672-7234513 & 11446.72 & -723451.3 & 11.37 & 0.3 & 12.67 & 0.55 & 1 & 1.81 & 40 & 3 & & $\ldots$ & $\ldots$ & $\ldots$ \\
\hline J01144979-7319179 & 11449.80 & $\begin{array}{ll}-12 & 4 \\
-73 & 18.0\end{array}$ & 11.49 & 0.38 & $\begin{array}{l}12.01 \\
12.99\end{array}$ & $\begin{array}{l}.00 \\
0.61\end{array}$ & 1 & $\begin{array}{l}1.01 \\
3.44\end{array}$ & 90.1 & 3 & F8V & $\ldots$ & $\ldots$ & \\
\hline J01145522-7309521 & 11455.23 & -730952.3 & 10.71 & 0.84 & 13.64 & 1.19 & 1 & 40.54 & 70.9 & 3 & & $\ldots$ & $\ldots$ & SkKM 332 \\
\hline J01151147-7308341 & 11511.48 & $-7308 \quad 34.2$ & 10.31 & 0.87 & 13.69 & 1.58 & 1 & 171.84 & 73.9 & 1 & .. & $\ldots$ & $\ldots$ & SkKM 333 \\
\hline J01151174-7355297 & 11511.72 & -735529.8 & 10.22 & 0.37 & 11.79 & 0.61 & 1 & -14.48 & 77.2 & 3 & & $\ldots$ & $\ldots$ & \\
\hline J01152452-7330443 & 11524.53 & -733044.4 & 10.48 & 0.43 & 12.08 & 0.63 & 1 & 181.24 & 52.5 & 1 & F2I & $\mathrm{F} 2$ & 6 & Flo 675 \\
\hline J01152857-7315370 & 11528.57 & $\begin{array}{llll}-73 & 15 & 37.1\end{array}$ & 12.25 & 0.15 & 12.82 & 0.11 & 1 & 161.74 & 35.5 & 1 & A3I & A3II & 3 & 2dFS 3094 \\
\hline J01153986-7221562 & 11539.88 & -722156.3 & 10.14 & 0.64 & 12.48 & 0.94 & 1 & 73.71 & 36.4 & 2 & & $\ldots$ & $\ldots$ & $\ldots$ \\
\hline J01155317-7330500 & 11553.18 & -733050.0 & $\begin{array}{r}1.14 \\
9.62\end{array}$ & 0.31 & 10.84 & $\ldots$ & 3 & -97.46 & $\begin{array}{l}0.4 \\
62.1\end{array}$ & 3 & hF5gF $2 \mathrm{mF} 0$ & $\mathrm{~F} 2$ & 16 & Flo 682 \\
\hline J01161289-7231363 & 11612.89 & -723136.3 & 9.82 & 0.45 & 11.56 & $\ldots$ & 3 & 30.71 & 99.9 & 3 & & G0 & 6 & Flo 685 \\
\hline J01161931-7252274 & 11619.30 & $-7252 \quad 27.5$ & 11.18 & 0.58 & 13.56 & 1.1 & 1 & 12.04 & 73.9 & 3 & K2III & .. & $\ldots$ & $\ldots$ \\
\hline J01165808-7311321 & 11658.10 & $\begin{array}{llll}-73 & 11 & 32.1\end{array}$ & 10.55 & 0.62 & 12.86 & 0.98 & 1 & 6.84 & 106.7 & 3 & KOIII & $\ldots$ & .. & SkKM 336 \\
\hline J01171108-7312214 & 11711.09 & $-7312 \quad 21.5$ & 11.42 & 0.41 & 13.21 & 0.8 & 1 & 7.94 & 92.3 & 3 & G5III & .. & .. & $\ldots$ \\
\hline J01171303-7317398 & $117 \quad 13.03$ & -731739.8 & 10.36 & 0.62 & 12.71 & 0.86 & 1 & 188.74 & 56.2 & 1 & G5I & $\ldots$ & $\ldots$ & $\ldots$ \\
\hline J01171843-7228088 & 11718.42 & -722808.8 & 11.09 & 0.2 & 12.01 & 0.4 & 1 & 12.31 & 37.1 & 3 & & B: & 6 & Flo 697 \\
\hline J01171937-7306068 & 11719.40 & -730606.8 & 11.18 & 0.31 & 12.45 & 0.5 & 1 & 2.34 & 108.2 & 3 & F5III: & ... & $\ldots$ & ... \\
\hline J01173565-7327031 & 11735.65 & -732703.2 & 9.39 & 0.64 & 11.8 & 0.95 & 1 & 172.64 & 82.3 & 1 & G9III & $\ldots$ & $\ldots$ & $\ldots$ \\
\hline J01175070-7315067 & 11750.73 & $\begin{array}{llll}-73 & 15 & 06.7\end{array}$ & 11.11 & 0.39 & $\begin{array}{r}12.0 \\
12.72\end{array}$ & 0.71 & 1 & 17.34 & 99.6 & 3 & GOIV & $\ldots$ & $\ldots$ & $\ldots$ \\
\hline J01175132-7316470 & 11751.34 & -731647.0 & 9.42 & 0.07 & 9.7 & $\ldots$ & 3 & -69.56 & 13.1 & 3 & $\mathrm{kA} 2 \mathrm{hA} 5 \mathrm{mFO}$ & $\mathrm{A} 2 \mathrm{~V}$ & 19 & HD 8096 \\
\hline J01175354-7321127 & 11753.56 & -732112.8 & 10.62 & 0.34 & 12 & 0.54 & 1 & 34.24 & 103 & 3 & F8V & ... & $\ldots$ & $\ldots$ \\
\hline J01180479-7324302 & 11804.79 & -732430.3 & 9.04 & 0.71 & 11.74 & 1.22 & 1 & 48.74 & 89.4 & 3 & KOIII & $\ldots$ & $\ldots$ & $\ldots$ \\
\hline J01182311-7324445 & 11823.12 & -732444.5 & 9.52 & 0.65 & 11.92 & 1.04 & 1 & -12.46 & 113.3 & 3 & KOIII & $\ldots$ & .. & $\ldots$ \\
\hline J01182380-7319158 & 11823.81 & -731915.8 & 9.73 & 0.72 & 12.35 & 1.16 & 1 & 23.94 & 114.1 & 3 & K2III & $\ldots$ & $\ldots$ & \\
\hline J01232375-7313342 & 12323.77 & $-7313 \quad 34.2$ & 9.67 & 0.67 & 12.16 & 1.14 & 1 & 17.98 & 88.8 & 3 & $\ldots$ & $\ldots$ & $\ldots$ & $\ldots$ \\
\hline J01234438-7332025 & 12344.37 & -733202.6 & 9.77 & 0.72 & 12.55 & $\begin{array}{l}1.14 \\
1.1\end{array}$ & 1 & -57.12 & 35.1 & 3 & & $\ldots$ & $\ldots$ & $\ldots$ \\
\hline J01240629-7314454 & 12406.29 & -731445.4 & 9.16 & 0.54 & 11.19 & $\ldots$ & 3 & 165.88 & 67.2 & 1 & $\ldots$ & F7I & 6 & Flo 739 \\
\hline J01243530-7313439 & 12435.30 & -731343.9 & 10.8 & 0.47 & 12.56 & 0.67 & 1 & 25.28 & 88.3 & 3 & & $\ldots$ & $\ldots$ & $\ldots$ \\
\hline J01255005-7323415 & 12550.06 & -732341.6 & 10.64 & 0.41 & 12.2 & 0.62 & 1 & 5.48 & 82.4 & 3 & $\ldots$ & $\ldots$ & $\ldots$ & \\
\hline J01260996-7323151 & 12609.98 & $-7323 \quad 15.1$ & 9.17 & 0.9 & 12.71 & 1.71 & 1 & 167.48 & 59.7 & 1 & $\ldots$ & $\ldots$ & $\ldots$ & SkKM 351 \\
\hline J01261633-7306310 & 12616.33 & -730630.9 & 11.67 & 0.26 & 12.71 & 0.45 & 1 & 27.38 & 46.8 & 3 & $\ldots$ & $\ldots$ & $\ldots$ & ... \\
\hline J01262548-7330334 & 12625.50 & -733033.4 & 10.67 & 0.65 & 13.04 & 0.98 & 1 & 50.68 & 36.4 & 3 & $\ldots$ & $\ldots$ & $\ldots$ & $\ldots$ \\
\hline J01272108-7314431 & 12721.10 & -731443.2 & 11.18 & 0.34 & 12.72 & 0.68 & 1 & 38.68 & 57.4 & 3 & $\ldots$ & $\ldots$ & $\ldots$ & $\ldots$ \\
\hline J01283670-7314496 & 12836.69 & -731449.6 & 11.56 & 0.31 & 12.81 & 0.51 & 1 & -2.12 & 37.7 & 3 & $\ldots$ & $\ldots$ & $\ldots$ & $\ldots$ \\
\hline J01290330-7334131 & 12903.30 & -733413.1 & 10.93 & 0.27 & 12.13 & 0.51 & 1 & 20.48 & 29.9 & 3 & & $\ldots$ & $\ldots$ & $\ldots$ \\
\hline J01303980-7322011 & 13039.81 & -732201.0 & 12.15 & 0.06 & 12.5 & 0.05 & 1 & 162.78 & 38.3 & 1 & & A2I: & 4 & Sk 185 \\
\hline
\end{tabular}

${ }^{\mathrm{a}}$ These stars were imaged twice and their results were averaged. The average difference in $\mathrm{Vel}_{\mathrm{obs}}$ was $2.2 \mathrm{~km} \mathrm{~s}^{-1}$ and the average difference in the $r$ parameter was 13.7 .

${ }^{\mathrm{b}}$ References for $B-V$ colors and $V$ magnitudes. $1=$ Massey 2002; $2=$ Mermilliod 1997; $3=$ ASAS-3; 4 = Zaritsky 2002; $5=($ Zaritsky B) $-($ ASAS-3 V)

${ }^{c}$ Tondry \& Davis $1979 r$ parameter. 
${ }^{\mathrm{d}}$ Category: 1 = SMC supergiant; $2=$ possible SMC supergiant; $3=$ foreground dwarf

${ }^{e}$ References for spectral classifications. $1=$ Cannon \& Pickering 1918; $2=$ Massey \& Olsen 2003; $3=$ Evans et al. 2004; $4=$ Sanduleak 1969; $5=$ Lü 1971; $6=$ Florsch 1972; $7=$ Lennon 1997; 8 = Azzopardi et al. 1975; 9 = Houk \& Cowley 1975; $10=$ Ardeberg \& Maurice 1977; $11=$ Azzopardi \& Vigneau 1979; $12=$ Gonzàlez-Fernàdez et al. $2015 ; 13=$ Humphreys 1983; $14=$ Humphreys et al. $1991 ; 15=$ Wallerstein $1984 ; 16=$ Bouchet et al. $1985 ; 17=$ Carney et al. $1985 ; 18=$ Sanduleak 1989

${ }^{\mathrm{f}}$ Comments include: alternate names for the star, comments on the blue spectra and comments based on the literature search. 
Table 4. Effective Temperatures Derived from ATLAS9 Models

\begin{tabular}{|c|c|c|c|c|c|c|c|}
\hline \multirow[b]{2}{*}{ Color } & \multirow[b]{2}{*}{ Observed } & \multicolumn{2}{|c|}{$E(B-V)=0.04$} & \multicolumn{2}{|c|}{$E(B-V)=0.09$} & \multicolumn{2}{|c|}{$E(B-V)=0.14$} \\
\hline & & Intrinsic & $T_{\text {eff }}(\mathrm{K})$ & Intrinsic & $T_{\text {eff }}(\mathrm{K})$ & Intrinsic & $T_{\text {eff }}(\mathrm{K})$ \\
\hline U-B & 0.78 & 0.75 & 4906 & 0.72 & 4943 & 0.68 & 4993 \\
\hline$B-V$ & 1.15 & 1.11 & 4768 & 1.06 & 4857 & 1.01 & 4945 \\
\hline $\mathrm{V}-\mathrm{K}$ & 2.94 & 2.82 & 4392 & 2.67 & 4499 & 2.53 & 4508 \\
\hline $\mathrm{J}-\mathrm{K}$ & 0.78 & 0.76 & 4394 & 0.73 & 4461 & 0.71 & 4508 \\
\hline Avg. & $\ldots$ & $\ldots$ & 4615 & $\ldots$ & 4690 & $\ldots$ & 4766 \\
\hline
\end{tabular}

Table 5. Physical Properties

\begin{tabular}{ll}
\hline \hline Parameter & Value \\
\hline Mass & $9 \mathrm{M}_{\odot}$ \\
Age & $30 \mathrm{Myr}$ \\
$\mathrm{T}_{\text {eff }}$ & $4700 \mathrm{~K}$ \\
$\log \mathrm{g}[\mathrm{cgs}]$ & 0.8 \\
$\mathrm{M}_{\mathrm{V}}$ & -5.4 \\
$\log \frac{\mathrm{L}}{\mathrm{L}_{\odot}}$ & 4.2 \\
Radius & $190 \mathrm{R}_{\odot}$ \\
\hline
\end{tabular}

